\title{
Functoriality in Resolution of Singularities
}

\author{
To Heisuke Hironaka for his 7rth birthday, \\ in celebration of Kiju - joy and long life!
}

By

Edward Bierstone* and Pierre D. Milman**

\begin{abstract}
Algorithms for resolution of singularities in characteristic zero are based on Hironaka's idea of reducing the problem to a simpler question of desingularization of an "idealistic exponent" (or "marked ideal"). How can we determine whether two marked ideals are equisingular in the sense that they can be resolved by the same blowing-up sequences? We show there is a desingularization functor defined on the category of equivalence classes of marked ideals and smooth morphisms, where marked ideals are "equivalent" if they have the same sequences of "test transformations". Functoriality in this sense realizes Hironaka's idealistic exponent philosophy. We use it to show that the recent algorithms for desingularization of marked ideals of Włodarczyk and of Kollár coincide with our own, and we discuss open problems.
\end{abstract}

\section{Contents}

$\S 1 . \quad$ Introduction

$\S 2 . \quad$ Marked Ideals and Test Transformations

$\S 3 . \quad$ Differential Calculus

$\S 4$. Maximal Contact and Coefficient Ideals

\footnotetext{
Communicated by S. Mori. Received February 13, 2007. Revised August 14, 2007, October 25, 2007.

2000 Mathematics Subject Classification(s): Primary 14E15, 32S45; Secondary 32S15.

Key words: resolution of singularities, functorial, canonical, marked ideal

The authors' research was supported by NSERC grants OGP0009070 and OGP0008949.

* Department of Mathematics, University of Toronto, Toronto, Ontario, Canada M5S 2E4.

e-mail: bierston@math.toronto.edu

** Department of Mathematics, University of Toronto, Toronto, Ontario, Canada M5S 2E4.

e-mail: milman@math.toronto.edu
}

(c) 2008 Research Institute for Mathematical Sciences, Kyoto University. All rights reserved. 
§5. Proof of Resolution of Singularities of a Marked Ideal

$\S 6$. Invariants of a Marked Ideal

§7. Passage from Local to Global

§8. Comparison of Algorithms and an Example

References

\section{$\S 1$. Introduction}

Hironaka's theorem on resolution of singularities in characteristic zero [Hi1, 1964] is one of the monuments of twentieth century mathematics. Canonical or functorial versions of Hironaka's theorem were established more than fifteen years ago, by Villamayor [V1], [V2] and by the authors [BM3], [BM4]. Two new treatments of canonical resolution of singularities have appeared just in the last couple of years, by Włodarcyzk [W] and Kollár [Ko]. These papers reflect a continuing interest in a better understanding of desingularization, in large part with the goal of discovering techniques that extend to positive characteristic (cf. [Hi3], [Hi4], [Ka]). They also show that certain aspects have remained mysterious, even in characteristic zero. Our goal here is to clarify these issues (and raise some questions).

The various proofs of desingularization have a lot in common, but also important differences that are related to the notion of functoriality or canonicity involved. They all involve reducing the problem to "canonical desingularization" of a collection of local resolution data, originating in Hironaka's idea of an idealistic exponent, and called a presentation [BM4], basic object [V2] or marked ideal $[\mathrm{W}]$ (with variations in the meaning of these objects). In all cases, canonical desingularization is proved by induction on dimension. Functoriality (apart from its intrinsic interest) plays two important roles: (i) in the statements of the theorems that can be proved as consequences of canonical desingularization of a marked ideal; (ii) in the proof itself - it is sometimes easier to prove a stronger theorem by induction, because we can make a stronger inductive assumption. (We use Włodarcyzk's terminology "marked ideal", though our notion is a little more general. See §1.1.)

We begin with the theorem of canonical resolution of singularities in characteristic zero (not the most general statement, but formulated in a way that is easy to state and includes the most useful conditions). For simplicity, we restrict our attention to algebraic varieties (or reduced separated schemes of finite type). (There are more general theorems for nonreduced schemes; see [Hi1], 
[BM4, Sect. 11]. Moreover, all results below apply to analytic spaces, where the desingularization morphism is given by a finite sequence of blowings-up over any relatively compact open set.)

Theorem 1.1. Given an algebraic variety $X$ over a field of characteristic zero, there is finite sequence of blowings-up $\sigma_{j}$ with smooth centres,

$$
X=X_{0} \stackrel{\sigma_{1}}{\longleftarrow} X_{1} \longleftarrow \cdots \stackrel{\sigma_{t}}{\longleftarrow} X_{t},
$$

such that:

(1) $X_{t}$ is smooth and the exceptional divisor $E_{t}$ in $X_{t}$ has only normal crossings.

(2) The morphism $\sigma$ given by the composite of the $\sigma_{j}$ is an isomorphism over $X \backslash$ Sing $X$.

(3) The resolution morphism $\sigma_{X}=\sigma: X_{t} \rightarrow X$ (or the entire sequence of blowings-up $\sigma_{j}$ ) can be associated to $X$ in a way that is functorial (at least with respect to étale or smooth morphisms, and field extensions).

This theorem can be proved with following stronger version of the condition (2): For each $j$, let $C_{j} \subset X_{j}$ denote the centre of the blowing-up $\sigma_{j+1}: X_{j+1} \rightarrow$ $X_{j}$, and let $E_{j}$ denote the exceptional divisor of $\sigma_{1} \circ \cdots \circ \sigma_{j}$. Then:

$\left(2^{\prime}\right)$ For each $j$, either $C_{j} \subset \operatorname{Sing} X_{j}$ or $X_{j}$ is smooth and $C_{j} \subset \operatorname{supp} E_{j}$.

Theorem 1.1 is proved in [BM4], in [Hi1] without the functoriality condition (3), and in [V2] assuming Hironaka's reduction to the case of an idealistic exponent [Hi2]. A weaker version of the theorem is proved in [EV2], [W], [Ko], where the blowings-up $\sigma_{j}$ do not necessarily have smooth centres. (We recall that every birational morphism of quasi-projective varieties is a blowing-up with centre that is not necessarily smooth [Ha, Thm. 7.17].) We do not know of a proof that provides the condition of smooth centres without also giving $\left(2^{\prime}\right)$. (See $\S 1.3$ below.)

There is no version of Theorem 1.1 that is functorial with respect to all morphisms. Consider the morphism $\varphi$ from $\mathbb{A}^{2}$ to the quadratic cone $X:=$ $\left\{u v-w^{2}=0\right\} \subset \mathbb{A}^{3}$ given by $\varphi(x, y)=\left(x^{2}, y^{2}, x y\right)[$ Ko, Ex. 3.4]. Then $\sigma_{\mathbb{A}^{2}}$ is the identity morphism (by functoriality with respect to translations), so functoriality with respect to $\varphi$ would imply that $\varphi$ lifts to the blowing-up $\sigma: X^{\prime} \rightarrow X$ over the origin (for example, by the universal mapping property of blowing up). But this is clearly false. 
The main subject of this article is functorial desingularization of a marked ideal ( $\$ 1.2$ befow); Theorem 1.3 is a strong version of functorial desingularization, proved in Sections 5-7. In $\$ 1.1$, we discuss how to deduce Theorem 1.1 from desingularization of a marked ideal. We show that, for $X$ embedded in a smooth variety $M$, the weaker version of Theorem 1.1 is an immediate consequence of desingularization of a marked ideal given essentially by the ideal of $X$ in $\mathcal{O}_{M}$. In $\S 1.3$, we indicate how Theorem 1.1 in full can be proved using our strong version of functorial desingularization for a different marked ideal that we call a presentation of the Hilbert-Samuel function of $X$; we refer to [BM4, Ch. III] for a construction of the latter.

\section{$\S 1.1$. Reduction to desingularization of a marked ideal}

For the sake of an inductive proof of Theorem 1.1, it is natural to deduce the result from an embedded version: We can assume that $X \subset M$, where $M$ is smooth. (Locally, $X$ can be embedded in an affine space.) Then the successive blowings-up of $X$ are given by restricting a sequence of blowings-up of $M$ to the corresponding strict transforms of $X$.

All currently available proofs of canonical embedded resolution of singularities follow Hironaka's idea of reducing the result to "functorial desingularization" of an ideal with associated multiplicity [Hi2]:

A marked ideal is a quintuple $\underline{\mathcal{I}}=(M, N, E, \mathcal{I}, d)$, where $N$ is a smooth subvariety of $M, E$ is a normal crossings divisor on $M$ that is transverse to $N$, $\mathcal{I}$ is a coherent ideal (sheaf) on $N$, and $d \in \mathbb{N}$. $\underline{\mathcal{I}}$ encodes the data we have at each step of resolution: $N$ will be a maximal contact subvariety and $E$ an exceptional divisor. A marked ideal has a transformation law that is simpler than strict transform: Say that a blowing-up $\sigma: M^{\prime} \rightarrow M$ is admissible if its centre $C \subset \operatorname{cosupp} \underline{\mathcal{I}}:=\left\{x \in N: \operatorname{order}_{x} \mathcal{I} \geq d\right\}$, and $C, E$ have only normal crossings. The (weak) transform $\mathcal{I}^{\prime}$ of $\mathcal{I}$ by $\sigma$ is generated by $y_{\text {exc }}^{-d} f \circ \sigma$, for all $f \in \mathcal{I}$, where $y_{\text {exc }}$ is a local generator of the ideal of $\sigma^{-1}(C)$. We define a resolution of singularities of $\underline{\mathcal{I}}$ as a finite sequence of admissible blowings-up, after which the cosupport is empty. (See Section 2 for detailed definitions.)

The definition of a marked ideal above is a slight generalization of that of [W] and corresponds to a presentation in [BM4]. Włodarczyk's marked ideal would be given by $\left(N,\left.E\right|_{N}, \mathcal{I}, d\right)$.

We prove desingularization of a marked ideal by induction on $\operatorname{dim} \underline{\mathcal{I}}$, where $\operatorname{dim} \underline{\mathcal{I}}:=\operatorname{dim} N$ : We reduce the dimension by passing to an equivalent coefficient ideal on a smooth maximal contact hypersurface. (See Defns. 2.5 and Sect. 4.) Maximal contact exists locally in characteristic zero, but is not unique. 
Coefficient ideals are defined using ideals of derivatives of $\mathcal{I}$ (The notion goes back to [Hi2], [G], and is used in [V2]). An idea in [BM4], [BM5] was to use derivatives preserving the exceptional divisor $E$ (i.e., derivatives logarithmic with respect to the exceptional variables). The idea is natural to the strong version of functoriality in Theorem 1.3 below (see §3.4), and is crucial to Kawanoue's proposal for desingularization in positive characteristic [Ka]. The use of derivatives preserving $E$ in resolution of singularities occurs already in $[\mathrm{C}]$.

There are at least two approaches to proving Theorem 1.1 using desingularization of a marked ideal. Given $X \subset M$, let $\mathcal{I}_{X} \subset \mathcal{O}_{M}$ denote the ideal of $X$. First, one can desingularize the marked ideal $\underline{\mathcal{I}}=\left(M, M, \emptyset, \mathcal{I}_{X}, 1\right)$ and restrict the sequence of blowings-up of $M$ that are involved to the successive strict transforms of $X$. This provides embedded resolution of singularities of $X$ in the case that $X \subset M$ is a hypersurface (i.e., of codimension 1), but only a weaker theorem in general (cf. [EV2], [W]): Consider the sequence of blowings-up $\left\{\sigma_{j}\right\}$ of $M$ that resolves the singularities of $\underline{\mathcal{I}}$ (and let $\underline{\mathcal{I}}_{j}$ denote the corresponding transforms of $\underline{\mathcal{I}}$ ). For some $r$, the centre $C_{r}$ of $\sigma_{r+1}$ coincides with $\operatorname{cosupp} \underline{\mathcal{I}}_{r}$. Then $X_{r}$ is smooth and has only normal crossings with $E_{r}$ (by Defns. 2.2). We get conditions (1)-(3) of Theorem 1.1, but the intersections $C_{j} \cap X_{j}$ are not necessarily smooth; i.e., the induced sequence of blowings-up of $X$ does not necessarily have smooth centres (nor do we necessarily get $C_{j} \cap X_{j} \subset \operatorname{Sing} X_{j}$ ). See Example 8.2.

To get Theorem 1.1 in full, we can follow a second approach (of origin in [Hi2]) which requires a stronger idea of functoriality in desingularization of a marked ideal (Thm. 1.3). This approach involves equisingularity of the centres of blowing up with respect to the Hilbert-Samuel function; Theorem 1.1 is obtained using Theorem 1.3 applied to a marked ideal which is constructed using a distinguished set of local generators of $\mathcal{I}_{X}$ [BM4, Ch. III]. We know of no other way to get Theorem 1.1 in full. (See $\S 1.3$ and Question 1.8.)

\section{§1.2. Test sequences and functorial desingularization}

Hironaka [Hi2] already proposed to deal with the preceding issue by using blowings-up not only as building blocks in resolution of singularities, but also to test for equisingularity of marked ideals. Define a weak test sequence for $\underline{I}$ as a sequence of morphisms, each either an admissible blowing-up or a projection $M \times \mathbb{A}^{1} \rightarrow M$. Say that two marked ideals on $M$ are weak equivalent if they have the same weak test sequences (Defns. 2.4).

Functoriality in a general sense should mean that "equivalent" marked ide- 
als undergo the same resolution process. In [Hi2, §9], Hironaka asserts (without proof) that a marked ideal can be desingularized functorially, not only with respect to smooth morphisms, but also with respect to weak equivalence classes (i.e., weak equivalent marked ideals have the same resolution sequences).

Question 1.2. Is this true?

The question is interesting partly in view of the role played by weak equivalence in [Hi3], which develops techniques for use in positive characteristic. We do not even know whether the algorithms of [BM4], [EV2], [W], [Ko] are functorial in the sense of Hironaka's assertion.

In [BM4], [BM5], we realize Hironaka's philosophy by enlarging the class of test transformations to include exceptional blowing-ups (centre $=$ an intersection of two components of the exceptional divisor). Say that two marked ideals on $M$ are equivalent if they have the same sequences of test transformations. (See Definitions 2.5.) The use of equivalence rather than weak equivalence is crucial to our proof of the following theorem - see Remarks 3.13 and 6.3.

Theorem 1.3 Functorial resolution of singularities of a marked ideal. Let $\underline{\mathcal{I}}=(M, N, E, \mathcal{I}, d)$ denote a marked ideal. Set $\underline{\mathcal{I}}_{0}:=\underline{\mathcal{I}}, \underline{\mathcal{I}}_{0}=\left(M_{0}, N_{0}, E_{0}\right.$, $\left.\mathcal{I}_{0}, d_{0}\right)$. Then there is a finite sequence of blowings-up with smooth centres,

$$
M=M_{0} \stackrel{\sigma_{1}}{\longleftarrow} M_{1} \longleftarrow \cdots \stackrel{\sigma_{t}}{\longleftarrow} M_{t},
$$

where $\sigma_{1}$ is admissible for $\underline{\underline{I}}_{0}$ and each successive $\sigma_{j+1}$ is admissible for the transform $\underline{\mathcal{I}}_{j}$ of $\underline{\underline{I}}_{j-1}$, such that

$$
\operatorname{cosupp} \underline{\mathcal{I}}_{t}=\emptyset .
$$

Moreover, a resolution sequence (1.1) can be associated to every marked ideal $\underline{\mathcal{I}}$ in a way that is functorial with respect to equivalence classes of marked ideals and smooth morphisms.

The functoriality assertion here means that, if $\underline{\mathcal{I}}=(M, N, E, \mathcal{I}, d), \underline{\mathcal{I}}_{1}=$ $\left(M_{1}, N_{1}, E_{1}, \mathcal{I}_{1}, d_{1}\right)$ are marked ideals and $\varphi: M_{1} \rightarrow M$ is a smooth morphism such that $\underline{I}_{1}$ and $\varphi^{*}(\underline{\mathcal{I}})$ are equivalent marked ideals of the same dimension, then $\varphi$ lifts to smooth morphisms throughout the resolution towers for $\underline{\mathcal{I}}_{1}, \underline{\mathcal{I}}$. Functoriality with respect to smooth morphisms in [W], $[\mathrm{Ko}]$ is the weaker assertion that $\varphi$ lifts to smooth morphisms throughout the resolution towers for $\varphi^{*}(\underline{\mathcal{I}})$ and $\underline{\mathcal{I}}$. When we say that $\varphi$ lifts to smooth morphisms throughout the resolution sequences, we allow the possibility of inserting trivial blowingsup (i.e., identity morphisms) in the resolution tower for $\underline{\mathcal{I}}_{1}$. (For example, if 
$\varphi: U \hookrightarrow M$ is an embedding of an open subvariety $U$, then the centre of a given blowing-up in the resolution sequence for $\underline{\mathcal{I}}$ may have no points over $U$.) This issue is treated in Section 7.

We give a complete proof of Theorem 1.3 in Sections 5-7. Our proof shows that the association of a resolution sequence to a marked ideal is also functorial with respect to field extensions and to closed embeddings $\iota: M \hookrightarrow M^{\prime}$ in the case that $d=1$ (as in [W], [Ko]), but we do not explicitly discuss these issues.

Our proof of Theorem 1.3 repeats the main features of [BM4] (and the corresponding algorithms are the same), but we use the very clear inductive framework of $[\mathrm{W}]$. Part of our purpose is to clarify the relationship between various proofs, particularly regarding the role of functoriality and the different notions of derivative ideal (Sect. 5). We also want to isolate the precise role of a desingularization invariant (Sect.7) and to show that the idea of equivalence makes it possible not only to prove the strong version of Theorem 1.1, but also to compare the actual recipes for choosing the centres of blowing up in different versions of desingularization of a marked ideal. In particular, we get the following result which we prove in $\S 8.1$ as a consequence of Corollaries 3.6, 5.3 and our proof of Theorem 1.3. (The coefficient ideal is defined in Section 4 (see also Sect. 5, Step I) and the companion ideal in Section 5, Step II.)

Corollary 1.4. The variants of the coefficient and companion ideals used in $[\mathrm{W}]$ or $[\mathrm{Ko}]$ are equivalent to our notions. As a result, the algorithm for resolution of singularities of marked ideals of Wtodarczyk or Kollár is essentially the same as that of [BM4] (see Remark 1.5(2) following).

Remark 1.5. (1) The various algorithms for resolution of singularities have a similar recursive structure. They differ essentially in the notions of coefficient and companion ideals that are used. These differences are reflected in important differences in the proofs of the canonical desingularization theorem, and one might ask whether the resulting algorithms provide different blowingup sequences - this is the issue treated in the second assertion of Corollary 1.4. We know of no way to prove this (or even to prove that the [W] and [Ko] algorithms coincide) other than using functoriality with respect to equivalence classes. See [BM5, §3.4] for the relationship between [BM4] and Villamayor's algorithm [V1], [V2].

(2) On the other hand, the desingularization algorithm (as described in Section 5) allows many minor variations which can be incorporated in any of the proofs of algorithmic resolution of singularities. For example: (a) Encinas and Villamayor's "good points" variant [EV1]; see [BM5, §3.6]. (b) Step I.B in Section 5 below (moving apart $E$ and $\operatorname{cosupp} \underline{\mathcal{I}}$ ) is described in a different 
way in [W] (and [Ko]) that may mean separating the components of $E$ from $\operatorname{cosupp} \underline{\mathcal{I}}$ in a somewhat different order. "Essentially the same" in Corollary 1.4 means that the algorithms of $[\mathrm{BM} 4]$ and $[\mathrm{W}]$ (or $[\mathrm{Ko}]$ ) coincide modulo the latter variation.

In Corollary 1.4 of course the blowings-up of $M$ have smooth centres; our discussion in $\S 1.1$ above concerns the way that desingularization of a marked ideal can be applied to prove Theorem 1.1.

Remark 1.6. There is another application of our notion of functoriality, raised by [Hi2, §1, Rmk. 2] and in an earlier version of [Ko]. Given two ideal sheaves one of which is integral over the other, it is easy to see that the associated marked ideals are equivalent, so that Theorem 1.3 associates the same resolution sequence to them (cf. [EV3]).

Theorem 1.3 is proved by induction on $\operatorname{dim} \underline{\mathcal{I}}$. (See Remark 1.7.) The inductive step breaks up into two distinct steps (Section 5):

I. Functorial desingularization of a marked ideal of dimension $n-1$ implies functorial desingularization of a marked ideal of maximal order, of dimension n. (See Definitions 2.1.)

II. Functorial desingularization of a marked ideal of maximal order of dimension $n$ implies functorial desingularization of a general marked ideal of dimension $n$.

Step I involves passing to an equivalent coefficient ideal on a local maximal contact hypersurface. We have to show that the centres of blowing up given by canonical desingularization of the coefficient ideals (which holds by induction) in overlapping coordinate charts, glue together to define a global centre of blowing up for the original marked ideal. Our proof and those of [W], [Ko] treat this problem in different ways: In our induction, the coefficient ideals in overlapping charts are equivalent, so that Step I follows from the inductive assumption of strong functorial desingularization in lower dimension - this is the reason for our notion of equivalence. But Step II requires some technical work. On the other hand, in $[\mathrm{W}],[\mathrm{Ko}]$, most of the work is required for Step I (passage to a "homogenized ideal", to reduce to a situation where maximal contact is uniquely determined up to an étale automorphism), while II is simpler.

Remark 1.7. The inductive assumption of functorial desingularization in lower dimension is used in our proof of Theorem 1.3 only in Step I, as just described. Functoriality with respect to smooth morphisms is an assertion which 
of course involves not only a fixed $\operatorname{dim} N$. We will give two different proofs that the locally defined resolution sequences for the coefficient ideals patch together to provide global blowings-up (Section 7). The first of these proofs uses only functoriality with respect to equivalence classes of marked ideals and particular étale morphisms (coverings by open charts) in the inductive assumption. The second uses only functoriality with respect to equivalence classes. With either approach, functoriality with respect to smooth morphisms follows using Lemmas 3.2 and 3.12 (see Remark 4.3).

\section{§1.3. Use of the Hilbert-Samuel function}

We can deduce Theorem 1.1 (including $\left(2^{\prime}\right)$ ) from Theorem 1.3, following Hironaka's philosophy in [Hi2]. We use the Hilbert-Samuel function

$$
H_{X, x}(k):=\text { length } \frac{\mathcal{O}_{X, x}}{\mathfrak{m}_{X, x}^{k+1}}, \quad k \in \mathbb{N} .
$$

The Hilbert-Samuel function $H_{X, .}: X \rightarrow \mathbb{N}^{\mathbb{N}}$ has the following basic properties,

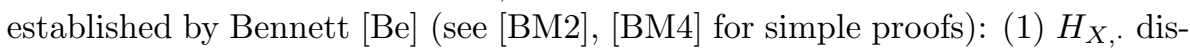
tinguishes smooth and singular points; (2) $H_{X}$, is Zariski upper-semicontinuous; (3) $H_{X}$, is infinitesimally upper-semicontinuous (i.e., $H_{X}$, cannot increase after blowing-up with centre on which it is constant); (4) Any decreasing sequence in the value set of the Hilbert-Samuel function stabilizes.

The Hilbert-Samuel function $H_{X}$, is a local invariant that plays the same role with respect to strict transform of a variety $X$ as the order with respect to (weak) transform of a (marked) ideal. More precisely, for all $a \in X$, there is a local embedding $X \hookrightarrow M$ at $a$ and a marked ideal $\underline{\mathcal{I}}=(M, N, \emptyset, \mathcal{I}, d)$ which has the same test sequences as $\underline{X}:=(M, \emptyset, X, H)$, where $H=H_{X, a}$. (We define a test sequence for $\underline{X}=(M, E, X, H)$ by analogy with that for a marked ideal (Defns. 2.5), but where a blowing-up $\sigma: M^{\prime} \rightarrow M$ with smooth centre $C$ is admissible if $C \subset \operatorname{supp} \underline{X}:=\left\{x \in X: H_{X, x} \geq H\right\}$ and $C, E$ have only normal crossings, and where $X$ transforms by strict transform.) We call $\underline{I}$ a presentation of $H_{X}$, at $a$. If $a$ is a maximum point of $H_{X, .}$, then the corresponding maximal value of $H_{X}$, decreases after desingularization of $\underline{\mathcal{I}}$, and Theorem 1.1 follows (using the basic properties of $H_{X}$, above and) using functoriality in the same way as in Step I of the proof of Theorem 1.3. (See [BM4, Ch. III] for these results.)

We are interested in equisingularity with respect to the Hilbert-Samuel function as a necessary rather than only sufficient condition on the centres of blowing up: 
Question 1.8. Does any local invariant $\iota_{X}$, which satisfies the basic properties (1)-(4) above and which admits a presentation determine the Hilbert-Samuel function $H_{X}$, ? For example, is the stratification of $X$ by the values of $\iota_{X}$, a refinement of that by $H_{X, \cdot}$ ?

\section{§2. Marked Ideals and Test Transformations}

In this section we give detailed definitions of the notions concerning marked ideals, test transformations and equivalence that have been introduced informally in Section 1.

Definitions 2.1. A marked ideal $\underline{\mathcal{I}}$ is a quintuple $\underline{\mathcal{I}}=(M, N, E, \mathcal{I}, d)$, where:

(1) $M, N$ are smooth, and $N \subset M$ is a closed subvariety;

(2) $E=\sum_{i=1}^{s} H_{i}$ is a simple normal crossings divisor on $M$ which is transverse to $N$ and ordered. (The $H_{i}$ are smooth hypersurfaces in $M$, not necessarily irreducible, with ordered index set as indicated.)

(3) $\mathcal{I}$ is an ideal (i.e., a coherent ideal sheaf) in $\mathcal{O}_{N}$.

(4) $d \in \mathbb{N}$.

We define the cosupport $\operatorname{cosupp} \underline{\mathcal{I}}$ of a marked ideal $\underline{\mathcal{I}}=(M, N, E, \mathcal{I}, d)$ as

$$
\operatorname{cosupp} \underline{\mathcal{I}}:=\left\{x \in N: \operatorname{ord}_{x} \mathcal{I} \geq d\right\},
$$

where $\operatorname{ord}_{x} \mathcal{I}$ denotes the largest nonnegative integer $q$ such that $\mathcal{I}_{x} \subset \mathfrak{m}_{N, x}^{q}$, with $\mathfrak{m}_{N, x}$ the maximal ideal of $\mathcal{O}_{N, x}$.

We say that a marked ideal $\underline{\mathcal{I}}=(M, N, E, \mathcal{I}, d)$ is of maximal order if $d=\max \left\{\operatorname{ord}_{x} \mathcal{I}: x \in \operatorname{cosupp} \underline{\mathcal{I}}\right\}$.

Definitions 2.2. Let $\underline{\mathcal{I}}=(M, N, E, \mathcal{I}, d)$ denote a marked ideal. A blowing-up $\sigma: M^{\prime} \rightarrow M$ (with smooth centre $C$ ) is admissible for $\underline{\mathcal{I}}$ if $C \subset$ $\operatorname{cosupp} \underline{\mathcal{I}}$, and $C, E$ have only normal crossings.

We define the transform of $\underline{\mathcal{I}}$ by an admissible blowing-up $\sigma: M^{\prime} \rightarrow M$ as the marked ideal $\underline{\mathcal{I}}^{\prime}=\left(M^{\prime}, N^{\prime}, E^{\prime}, \mathcal{I}^{\prime}, d^{\prime}=d\right)$, where:

(1) $N^{\prime}$ is the strict transform of $N$ by $\sigma$.

(2) $E^{\prime}=\sum_{i=1}^{s+1} H_{i}^{\prime}$, where $H_{i}^{\prime}$ denotes the strict transform of $H_{i}$, for each $i=1, \ldots, s$, and $H_{s+1}^{\prime}:=\sigma^{-1}(C)$ (the exceptional divisor of $\sigma$, introduced as the last member of $E^{\prime}$ ). 
(3) $\mathcal{I}^{\prime}:=\mathcal{I}_{\sigma^{-1}(C)}^{-d} \cdot \sigma^{*}(\mathcal{I})\left(\right.$ where $\mathcal{I}_{\sigma^{-1}(C)} \subset \mathcal{O}_{N^{\prime}}$ denotes the ideal of $\sigma^{-1}(C)$ ). I.e., $\mathcal{I}^{\prime}$ is generated locally by $y_{\text {exc }}^{-d} f \circ \sigma$, for all local generators $f$ of $\mathcal{I}$, where $y_{\text {exc }}$ denotes a local generator of $\mathcal{I}_{\sigma^{-1}(C)}$.

In this definition, note that $\sigma^{*}(\mathcal{I})$ is divisible by $\mathcal{I}_{\sigma^{-1}(C)}^{d}$ and $E^{\prime}$ is a normal crossings divisor transverse to $N^{\prime}$, because $\sigma$ is admissible.

Definition 2.3. Let $\underline{\mathcal{I}}=(M, N, E, \mathcal{I}, d)$ denote a marked ideal, and let $\varphi: M_{1} \rightarrow M$ be a smooth morphism. We define the pull-back $\varphi^{*} \underline{\mathcal{I}}$ of $\underline{\mathcal{I}}$ as the marked ideal $\varphi^{*} \underline{\mathcal{I}}:=\left(M_{1}, \varphi^{-1}(N), \varphi^{-1}(E), \varphi^{*}(\mathcal{I}), d\right)$.

Definitions 2.4. Let $\underline{\mathcal{I}}=(M, N, E, \mathcal{I}, d)$ denote a marked ideal as above. Consider the product of $M$ with a line, $M^{\prime}:=M \times \mathbb{A}^{1}$, and let $\pi: M^{\prime} \rightarrow M$ denote the projection morphism. We define the transform $\underline{\mathcal{I}}^{\prime}$ of $\underline{\mathcal{I}}$ by $\pi$ as the marked ideal $\underline{\mathcal{I}}^{\prime}=\left(M^{\prime}, N^{\prime}, E^{\prime}, \mathcal{I}^{\prime}, d^{\prime}=d\right)$, where $N^{\prime}:=\pi^{-1}(N)$, $\mathcal{I}^{\prime}:=\pi^{*}(\mathcal{I})$, and $E^{\prime}=\sum_{i=1}^{s+1} H_{i}^{\prime}$, where $H_{i}^{\prime}:=\pi^{-1}\left(H_{i}\right)$, for each $i=1, \ldots, s$, and $H_{s+1}^{\prime}$ denotes the horizontal divisor $D:=M \times\{0\}$ (included as the last member of $E^{\prime}$ ). (Of course, $\pi$ is a smooth morphism, but $\underline{\mathcal{I}}^{\prime}$ differs from $\pi^{*}(\underline{\mathcal{I}})$ in the term $E^{\prime}$.)

We define a weak test sequence for $\underline{\mathcal{I}}_{0}=\underline{\mathcal{I}}$ as a sequence of morphisms

$$
M=M_{0} \stackrel{\sigma_{1}}{\longleftarrow} M_{1} \longleftarrow \cdots \stackrel{\sigma_{t}}{\longleftarrow} M_{t},
$$

where each successive morphism $\sigma_{j+1}$ is either an admissible blowing-up (for the transform $\underline{\mathcal{I}}_{j}$ of $\underline{\mathcal{I}}_{j-1}$ ), or the projection from the product with a line.

We say that two marked ideals $\underline{\mathcal{I}}$ and $\underline{\mathcal{I}}_{1}=\left(M_{1}=M, N_{1}, E_{1}=E, \mathcal{I}_{1}, d_{1}\right)$ (with the same ambient variety $M$ and the same normal crossings divisor $E$ ) are weak-equivalent if they have the same weak test sequences; i.e., every weak test sequence for one is a weak test sequence for the other.

Definitions 2.5. $\quad$ Let $\underline{\mathcal{I}}=(M, N, E, \mathcal{I}, d)$ be a marked ideal. A blowingup $\sigma: M^{\prime} \rightarrow M$ will be called an exceptional blowing-up for $\mathcal{I}$ if its centre $C$ is an intersection $H_{i} \cap H_{j}$ of distinct hypersurfaces $H_{i}, H_{j} \in E$.

We define the transform $\underline{\mathcal{I}}^{\prime}$ of $\underline{\mathcal{I}}$ by an exceptional blowing-up $\sigma: M^{\prime} \rightarrow M$ (with centre $C$, say) as the marked ideal $\underline{\mathcal{I}}^{\prime}=\left(M^{\prime}, N^{\prime}, E^{\prime}, \mathcal{I}^{\prime}, d^{\prime}\right)$, where $N^{\prime}$ the strict transform of $N\left(N^{\prime}=\sigma^{-1}(N)\right.$, in this case), $E^{\prime}$ is defined as in Definitions 2.2 above, $\mathcal{I}^{\prime}=\sigma^{*}(\mathcal{I})$, and $d^{\prime}=d$.

We define a test sequence for $\underline{\mathcal{I}}_{0}=\underline{\mathcal{I}}$ as a sequence of morphisms (2.1), where each successive $\sigma_{j+1}$ is either an admissible blowing-up, the projection 
from a product with a line, or an exceptional blowing-up. (Morphisms of these three kinds will be called test transformations or test morphisms.)

We say that two marked ideals $\underline{\mathcal{I}}$ and $\underline{\mathcal{I}}_{1}$ (with the same ambient variety $M$ and the same normal crossings divisor $E$ ) are equivalent if they have the same test sequences.

\section{§3. Differential Calculus}

Let $N$ denote a smooth variety. If $\left(x_{1}, \ldots, x_{n}\right)$ are local coordinates at a point of $N$, then the partial derivatives $\partial / \partial x_{1}, \ldots, \partial / \partial x_{n}$ are well-defined as local generators of the sheaf of derivations $\operatorname{Der}_{N}$ of $\mathcal{O}_{N}$.

\section{§3.1. Transformation of differential operators by blowing up}

Let $\sigma: N^{\prime} \rightarrow N$ denote the blowing-up with centre a smooth subvariety $C$ of $N$. Choose a local coordinate chart $U$ of $N$ with coordinates $\left(x_{1}, \ldots, x_{n}\right)$ in which $C=\left\{x_{r}=\cdots=x_{n}=0\right\}$. Then $\sigma^{-1}(C)$ is covered by coordinate charts $U_{x_{r}}, \ldots, U_{x_{n}}$, where, for example in the $x_{r}$-chart $U_{x_{r}}$, we can choose coordinates $\left(y_{1}, \ldots, y_{n}\right)$ with $y_{1}=x_{1}, \ldots, y_{r}=x_{r}, y_{r+1}=x_{r+1} / x_{r}, \ldots, y_{n}=x_{n} / x_{r}$. The following lemma is a simple but crucial exercise using the chain rule.

Lemma 3.1. With coordinates chosen as above, we have:

$$
\begin{gathered}
\frac{\partial}{\partial x_{j}}=\frac{\partial}{\partial y_{j}}, \quad j=1, \ldots, r-1, \\
x_{r} \frac{\partial}{\partial x_{r}}=y_{r} \frac{\partial}{\partial y_{r}}-\sum_{j=r+1}^{n} y_{j} \frac{\partial}{\partial y_{j}}, \\
x_{j} \frac{\partial}{\partial x_{j}}=y_{j} \frac{\partial}{\partial y_{j}}, \quad j=r+1, \ldots, n .
\end{gathered}
$$

The transformation formulas in Lemma 3.1 go back to [G], and are usually written a little differently. As arranged here, they show that it is logarithmic differential operators which transform naturally by blowing up.

\section{§3.2. Derivative ideals}

Let $\mathcal{I} \subset \mathcal{O}_{N}$ denote a (coherent) ideal. Let $\mathcal{D}(\mathcal{I}) \subset \mathcal{O}_{N}$ denote the coherent sheaf of ideals generated by all first derivatives of local sections of $\mathcal{I}$ (so that $\mathcal{I} \subset \mathcal{D}(\mathcal{I})$ ); i.e., $\mathcal{D}(\mathcal{I})$ is the image of the natural morphism $\operatorname{Der}_{N} \times \mathcal{I} \rightarrow \mathcal{O}_{N}$. 
If $f_{1}, \ldots, f_{q}$ are local generators of $\mathcal{I}$ in a chart with coordinates $\left(x_{1}, \ldots\right.$, $\left.x_{n}\right)$, then $f_{i}, \partial f_{i} / \partial x_{j}$, where $i=1, \ldots, q, j=1, \ldots, n$, are local generators of $\mathcal{D}(\mathcal{I})$.

We also define higher-derivative ideals inductively by

$$
\mathcal{D}^{j+1}(\mathcal{I}):=\mathcal{D}\left(\mathcal{D}^{j}(\mathcal{I})\right), \quad j=1, \ldots
$$

Now let $E$ denote a normal-crossings divisor on $N$. We define $\mathcal{D}_{E}(\mathcal{I}) \subset \mathcal{O}_{N}$ as the ideal generated by all local sections of $\mathcal{I}$ and first derivatives which preserve the ideal $\mathcal{I}_{E}$ of $E$. (If $N \subset M$, where $M$ is smooth, and $E$ is a normalcrossings divisor on $M$ which is transverse to $N$, then we will simply write $\mathcal{D}_{E}(\mathcal{I})$ instead of $\mathcal{D}_{\left.E\right|_{N}}(\mathcal{I})$.)

Consider a divisor $E=\sum_{i=1}^{r} \alpha_{i} H_{i}$, where each $\alpha_{i} \in \mathbb{N}$. Each point of $N$ has a neighbourhood $U$ with a coordinate system $\left(x_{1}, \ldots, x_{q}, \ldots, x_{n}\right), q \leq r$, such that $x_{1}, \ldots, x_{q}$ generate the ideals $\left.\mathcal{I}_{H}\right|_{U}$ for those $H=H_{i}$ which intersect $U$; write $x_{j}=x_{H}$, where $H$ is the corresponding divisor. If $f_{i}$ are local generators of $\mathcal{I}$, then $\mathcal{D}_{E}(\mathcal{I})$ is generated locally by all

$$
f_{i}, \quad x_{H} \frac{\partial f_{i}}{\partial x_{H}}, \quad \text { and } \quad \frac{\partial f_{i}}{\partial x_{j}}, \quad q+1 \leq j \leq n .
$$

We again define

$$
\mathcal{D}_{E}^{j+1}(\mathcal{I}):=\mathcal{D}_{E}\left(\mathcal{D}_{E}^{j}(\mathcal{I})\right), \quad j=1, \ldots
$$

Now consider a marked ideal $\underline{\mathcal{I}}=(M, N, E, \mathcal{I}, d)$. For simplicity, we will write $\underline{\mathcal{I}}=(\mathcal{I}, d)$ when the remaining entries are evident. We define

$$
\begin{aligned}
\underline{\mathcal{D}}(\underline{\mathcal{I}}) & :=(M, N, E, \mathcal{D}(\mathcal{I}), d-1)=(\mathcal{D}(\mathcal{I}), d-1), \\
\underline{\mathcal{D}}_{E}(\underline{\mathcal{I}}) & :=\left(M, N, E, \mathcal{D}_{E}(\mathcal{I}), d-1\right)=\left(\mathcal{D}_{E}(\mathcal{I}), d-1\right) .
\end{aligned}
$$

and

$$
\underline{\mathcal{D}}_{E}^{j+1}(\underline{\mathcal{I}}):=\underline{\mathcal{D}}_{E}\left(\underline{\mathcal{D}}_{E}^{j}(\underline{\mathcal{I}})\right)=\left(\mathcal{D}_{E}^{j+1}(\mathcal{I}), d-j-1\right), \quad j=1, \ldots, d-1 .
$$

Lemma 3.2. Let $\underline{\mathcal{I}}=(M, N, E, \mathcal{I}, d)$ be a marked ideal. Then:

(1) $\mathcal{D}_{E}^{k}\left(\mathcal{D}_{E}^{l}(\mathcal{I})\right)=\mathcal{D}_{E}^{k+l}(\mathcal{I})$.

(2) $\mathcal{D}_{E}^{k}(\mathcal{I} \cdot \mathcal{J}) \subset \sum_{j=0}^{k} \mathcal{D}_{E}^{j}(\mathcal{I}) \cdot \mathcal{D}_{E}^{k-j}(\mathcal{J})$.

(3) $\operatorname{cosupp} \underline{\mathcal{I}} \subset \operatorname{cosupp} \underline{\mathcal{D}}_{E}^{j}(\underline{\mathcal{I}}), j=0,1, \ldots, d-1$, with equality if $E=\emptyset$.

(4) If $\varphi: P \rightarrow N$ is a smooth morphism, then $\mathcal{D}_{\varphi^{-1}(E)}\left(\varphi^{*}(\mathcal{I})\right)=\varphi^{*}\left(\mathcal{D}_{E}(\mathcal{I})\right)$. 
Proof. These statements are immediate from the definitions. (It is enough to check (4) on completions.)

Lemma 3.3. Let $\underline{\mathcal{I}}=(M, N, E, \mathcal{I}, d)$ be a marked ideal. Let $\sigma: M^{\prime} \rightarrow$ $M$ denote a test transformation for $\underline{\mathcal{I}}$ (i.e., either an admissible or exceptional blowing-up, or a projection $\left.M \times \mathbb{A}^{1} \rightarrow M\right)$, and let $\underline{\mathcal{I}}^{\prime}=\left(M^{\prime}, N^{\prime}, E^{\prime}, \mathcal{I}^{\prime}, d^{\prime}\right)$ denote the transform of $\underline{\mathcal{I}}$ by $\sigma$. (See Sect. 2.) Then $\sigma$ is a test transformation for $\underline{\mathcal{D}}_{E}(\underline{\mathcal{I}})$, and $\mathcal{D}_{E}(\mathcal{I})^{\prime} \subset \mathcal{D}_{E^{\prime}}\left(\mathcal{I}^{\prime}\right)$.

Proof. The first assertion follows from Lemma 3.2(3), and the second is an exercise using the transformation formulas of Lemma 3.1.

The following three corollaries are easy consequences of Lemma 3.3.

Corollary 3.4. With the hypotheses of Lemma 3.3, if $j \leq d-1$, then $\mathcal{D}_{E}^{j}(\mathcal{I})^{\prime} \subset \mathcal{D}_{E^{\prime}}^{j}\left(\mathcal{I}^{\prime}\right)$.

Corollary 3.5. If $j \leq d-1$, then any sequence of test transformations of $\underline{\mathcal{I}}$ is a sequence of test transformations of $\underline{\mathcal{D}}_{E}^{j}(\underline{\mathcal{I}})$ and, after any sequence of test transformations

$$
M=M_{0} \stackrel{\sigma_{1}}{\longleftarrow} M_{1} \longleftarrow \cdots \stackrel{\sigma_{t}}{\longleftarrow} M_{t}
$$

we have an inclusion $\mathcal{D}_{E}^{j}(\mathcal{I})_{t} \subset \mathcal{D}_{E_{t}}^{j}\left(\mathcal{I}_{t}\right)$.

Of course, we can also define $\underline{\mathcal{D}}^{j+1}(\underline{\mathcal{I}}), j=1, \ldots$, by iterating $\underline{\mathcal{D}}$, and we have analogues of Lemmas 3.2, 3.3 and Corollaries 3.4, 3.5 for the $\underline{\mathcal{D}}^{j}(\underline{\mathcal{I}})$. We will use the following to prove Corollary 1.4.

Corollary 3.6. Suppose that $E=\emptyset$. Under the hypotheses of Lemma 3.3 , if $j \leq d-1$, then $\mathcal{D}^{j}(\mathcal{I})^{\prime} \subset \mathcal{D}_{E^{\prime}}^{j}\left(\mathcal{I}^{\prime}\right)$. Likewise, after any sequence of test transformations as in Corollary $3.5, \mathcal{D}^{j}(\mathcal{I})_{t} \subset \mathcal{D}_{E_{t}}^{j}\left(\mathcal{I}_{t}\right)$.

\section{§3.3. Aside on sums and products of marked ideals}

We define sums and products, and give two lemmas which are simple exercises (cf. [W, Lemma 3.4.1]).

Consider marked ideals $\underline{\mathcal{I}}=(M, N, E, \mathcal{I}, d)=(\mathcal{I}, d)$ and $\underline{\mathcal{J}}=(M, N, E, \mathcal{J}, d)$ $=(\mathcal{J}, d)$. Define $\underline{\mathcal{I}} \cdot \underline{\mathcal{J}}:=(\mathcal{I} \cdot \mathcal{J}, d+e)$. 


\section{Lemma 3.7.}

(1) $\operatorname{cosupp} \underline{\mathcal{I}} \cap \operatorname{cosupp} \underline{\mathcal{J}} \subset \operatorname{cosupp} \underline{\mathcal{I}} \cdot \underline{\mathcal{J}}\left(\right.$ whereas $\operatorname{cosupp} \underline{\mathcal{I}}^{k}=\operatorname{cosupp} \underline{\mathcal{I}}$.)

(2) Let $\sigma: M^{\prime} \rightarrow M$ be a test morphism for both $\underline{\mathcal{I}}$ and $\underline{\mathcal{I}}$. Then $\sigma$ is a test morphism for $\underline{\mathcal{I}} \cdot \underline{\mathcal{J}}$ and the transforms satisfy $\underline{\mathcal{I}}^{\prime} \cdot \underline{\mathcal{J}^{\prime}}=(\underline{\mathcal{I}} \cdot \underline{\mathcal{J}})^{\prime}$.

(3) Multiplication of marked ideals is associative.

Define $\underline{\mathcal{I}}+\underline{\mathcal{J}}:=\left(\underline{\mathcal{I}}^{l / d}+\underline{\mathcal{J}}^{l / e}, l\right)$, where $l=\operatorname{lcm}(d, e)$. Likewise, for any finite sum. Addition is not associative, but $\underline{\mathcal{I}}+\underline{\mathcal{J}}$ is equivalent to $\left(\underline{\mathcal{I}}^{e}+\underline{\mathcal{J}}^{d}, d e\right)$. (See Lemma 3.8(3).)

\section{Lemma 3.8.}

(1) $\operatorname{cosupp}(\underline{\mathcal{I}}+\underline{\mathcal{J}})=\operatorname{cosupp} \underline{\mathcal{I}} \cap \operatorname{cosupp} \underline{\mathcal{J}}$.

(2) $\sigma: M^{\prime} \rightarrow M$ is a test morphism for $\underline{\mathcal{I}}+\underline{\mathcal{J}}$ if and only if $\sigma$ is a test morphism for both $\underline{\mathcal{I}}$ and $\underline{\mathcal{J}}$, and the transforms by such a test morphism satisfy $\underline{\mathcal{I}}^{\prime}+\underline{\mathcal{J}}^{\prime}=(\underline{\mathcal{I}}+\underline{\mathcal{J}})^{\prime}$.

(3) Addition of marked ideals is associative up to equivalence.

(4) If either $\underline{\mathcal{I}}$ or $\underline{\mathcal{J}}$ has maximal order and $\operatorname{cosupp}(\underline{\mathcal{I}}+\underline{\mathcal{J}}) \neq \emptyset$, then $\underline{\mathcal{I}}+\underline{\mathcal{J}}$ has maximal order.

\section{§3.4. Logarithmic derivatives determine equivalent ideals}

Consider a marked ideal $\underline{\mathcal{I}}=(M, N, E, \mathcal{I}, d)$. We define

$$
\underline{\mathcal{C}}_{E}^{k}(\underline{\mathcal{I}}):=\sum_{j=0}^{k} \underline{\mathcal{D}}_{E}^{j}(\underline{\mathcal{I}}), \quad k \leq d-1 .
$$

Write $\underline{\mathcal{C}}_{E}^{k}(\underline{\mathcal{I}})=\left(M, N, E, \mathcal{C}_{E}^{k}(\underline{\mathcal{I}}), d_{\underline{\mathcal{C}}_{E}^{k}(\underline{\mathcal{I}})}\right)$

Lemma 3.9. $\operatorname{cosupp} \underline{\mathcal{I}}=\operatorname{cosupp} \underline{\mathcal{C}}_{E}^{k}(\underline{\mathcal{I}})$.

Proof. $\quad \operatorname{cosupp} \underline{\mathcal{I}} \subset \operatorname{cosupp} \underline{\mathcal{C}}_{E}^{k}(\underline{\mathcal{I}})$, by Lemmas 3.2(3) and 3.8(1). But $\operatorname{cosupp} \underline{\mathcal{C}}_{E}^{k}(\underline{\mathcal{I}}) \subset \operatorname{cosupp} \underline{\mathcal{I}}$, since $\underline{\mathcal{D}}_{E}^{0}(\underline{\mathcal{I}})=\underline{\mathcal{I}}$.

Theorem 3.10. Consider any sequence of test morphisms for $\underline{\mathcal{I}}$,

$$
M=M_{0} \stackrel{\sigma_{1}}{\longleftarrow} M_{1} \longleftarrow \cdots \stackrel{\sigma_{t}}{\longleftarrow} M_{t} .
$$

Then (3.1) is a sequence of test morphisms for $\underline{\mathcal{C}}_{E}^{k}(\underline{\mathcal{I}})$, and for the transforms, we have

$$
\operatorname{cosupp} \underline{\mathcal{C}}_{E_{t}}^{k}\left(\underline{\mathcal{I}}_{t}\right)=\operatorname{cosupp} \underline{\mathcal{C}}_{E}^{k}(\underline{\mathcal{I}})_{t}
$$


Proof. By Corollary 3.5, any sequence of test morphisms of $\underline{\mathcal{I}}$ is a sequence of test morphisms of $\underline{\mathcal{C}}_{E}^{k}(\underline{\mathcal{I}})$, and $\operatorname{cosupp} \underline{\mathcal{C}}_{E_{t}}^{k}\left(\underline{\mathcal{I}}_{t}\right) \subset \operatorname{cosupp} \underline{\mathcal{C}}_{E}^{k}(\underline{\mathcal{I}})_{t}$. Since $\underline{\mathcal{C}}_{E}^{k}(\underline{\mathcal{I}})=\underline{\mathcal{I}}+\sum_{j=1}^{k} \underline{\mathcal{D}}_{E}^{j}(\underline{\mathcal{I}})$, we have

$$
\begin{aligned}
\operatorname{cosupp} \underline{\mathcal{C}}_{E}^{k}(\underline{\mathcal{I}})_{t} & \subset \operatorname{cosupp} \underline{\mathcal{I}}_{t} \\
& \subset \bigcap_{j=0}^{k} \operatorname{cosupp} \underline{\mathcal{D}}_{E_{t}}^{j}\left(\underline{\mathcal{I}}_{t}\right), \quad \text { by Lemma } 3.2(3) \\
& =\operatorname{cosupp} \underline{\mathcal{C}}_{E_{t}}^{k}\left(\underline{\mathcal{I}}_{t}\right)
\end{aligned}
$$

Corollary 3.11. $\quad \underline{\mathcal{I}}$ and $\underline{\mathcal{C}}_{E}^{k}(\underline{\mathcal{I}})$ are equivalent.

Proof. They have the same test sequences, by Lemma 3.9 and Theorem 3.10 .

Lemma 3.12. If $\varphi: M^{\prime} \rightarrow M$ is smooth, then $\varphi^{*}\left(\underline{\mathcal{C}}_{E}^{k}(\underline{\mathcal{I}})\right)=$ $\underline{\mathcal{C}}_{\varphi^{-1}(E)}^{k}\left(\varphi^{*}(\underline{\mathcal{I}})\right)$.

Proof. This follows from Lemma 3.2(4).

Remark 3.13. The objects constructed in this section and the following using our logarithmic derivative ideals $\underline{\mathcal{D}}_{E}^{j}(\underline{\mathcal{I}})$ of course have analogues defined using the standard derivative ideals $\underline{\mathcal{D}}^{k}(\underline{\mathcal{I}})$ (cf. [V2]): We define $\underline{\mathcal{C}}^{k}(\underline{\mathcal{I}}):=$ $\sum_{j=0}^{k} \underline{\mathcal{D}}^{j}(\underline{\mathcal{I}}), k \leq d-1$. Then $\underline{\mathcal{I}}$ and $\underline{\mathcal{C}}^{k}(\underline{\mathcal{I}})$ are weak-equivalent (cf. Corollary 3.11 ), but in general they are not equivalent. Step II of our proof of Theorem 1.3 is not functorial with respect to weak equivalence classes.(See Remark 6.3 and Question 1.2.) We need the logarithmic derivative ideals to prove functoriality with respect to equivalence classes, using Theorems 6.1 and 6.2 and Corollary 5.3 .

\section{$\S 4$. Maximal Contact and Coefficient Ideals}

Corollary 4.1. Let $\underline{\mathcal{I}}=(M, N, E, \mathcal{I}, d)$ denote a marked ideal. Let $z$ denote a section of $\mathcal{D}_{E}^{d-1}(\mathcal{I})$ on $\left.N\right|_{U}$, where $U$ is an open subset of $M$. Suppose that $z$ has maximum order 1 and that $z$ is transverse to $E$ (equivalently, $\left.z \cdot \mathcal{I}_{E}\right|_{N}$ has only normal crossings and $\left.\mathcal{I}_{E}\right|_{N} \not \subset(z)$, where $\left.(z) \subset \mathcal{O}_{N}\right|_{U}$ is the ideal generated by $z)$. Let $P=V(z)$. Then, after any sequence of test transformations of $\left.\underline{\mathcal{I}}\right|_{U}$

$$
\operatorname{cosupp} \underline{\mathcal{I}}_{t} \subset P_{t} \subset N_{t}
$$


where $P_{t}=V\left((z)_{t}\right)$ and $(z)_{t}$ denotes the transform of $(z)$ (cf. notation of Thm.3.10). Moveover, if $\operatorname{cosupp} \underline{\mathcal{I}} \subsetneq P$, then $(z)_{t}$ has maximum order 1 and is transverse to $E_{t}$.

Proof. This follows from Theorem 3.10 and Corollary 3.11.

In the situation of Corollary 4.1, we call the hypersurface $P$ of $\left.N\right|_{U}$ a hypersurface of maximal contact for $\underline{\mathcal{I}}$. Note that the existence of $z$ with maximum order 1 means that $\underline{I}$ is of maximal order on $U$. In the setting of Corollary 4.1, we define the coefficient (marked) ideal

$$
\underline{\mathcal{C}}_{E, P}(\underline{\mathcal{I}}):=\left(U, P, E,\left.\mathcal{C}_{E}^{d-1}(\underline{\mathcal{I}})\right|_{P}, d_{\underline{\mathcal{C}}_{E}^{d-1}(\underline{\mathcal{I}})}\right) .
$$

The following is an immediate consequence of Corollary 4.1.

Corollary 4.2. $\quad \underline{\mathcal{C}}_{E, P}(\underline{\mathcal{I}})$ is equivalent to $\left.\underline{\underline{I}}\right|_{U}$.

Remark 4.3. $\underline{\mathcal{C}}_{E, P}$ commutes with smooth morphisms. In other words, if $\varphi: M^{\prime} \rightarrow M$ is smooth, then $P^{\prime}:=\varphi^{-1}(P)=V\left(\varphi^{*}(z)\right)$ is a maximal contact hypersurface for $\varphi^{*}(\underline{\mathcal{I}})$, and $\varphi^{*}\left(\underline{\mathcal{C}}_{E, P}(\underline{\mathcal{I}})\right)=\underline{\mathcal{C}}_{E^{\prime}, P^{\prime}}\left(\varphi^{*}(\underline{\mathcal{I}})\right)$ (by Lemma 3.12).

Exercises 4.4. The following exercises give alternative ways to define coefficient ideals. These approaches are used in [BM2], [BM4], and can simplify explicit calculations.

(1) Under the hypotheses of Corollary 4.1, we can find a system of local coordinates $\left(x_{1}, \ldots, x_{n}\right)$ for $N$ such that $x_{n}=z$ and the components of $E$ are given by $x_{i}=0, i=1, \ldots, r<n$ (in this chart). Let $\mathcal{D}_{z}(\mathcal{I})$ denote the ideal generated by $f, \partial f / \partial z$, for all $f \in \mathcal{I}$ (so that $\mathcal{I} \subset \mathcal{D}_{z}(\mathcal{I}) \subset \mathcal{D}_{E}(\mathcal{I})$ ), and set $\underline{\mathcal{C}}_{z}^{d-1}(\underline{\mathcal{I}}):=\sum_{j=0}^{d-1} \underline{\mathcal{D}}_{z}^{j}(\underline{\mathcal{I}})$. Then $\underline{\mathcal{C}}_{z, P}(\underline{\mathcal{I}}):=\left(U, P, E,\left.\mathcal{C}_{z}^{d-1}(\underline{\mathcal{I}})\right|_{P}, d_{\underline{\mathcal{C}}_{z}^{d-1}(\underline{\mathcal{I}})}\right)$ is equivalent to $\underline{\mathcal{I}}$ (in the chart).

(2) Suppose that $\mathcal{I} \subset \mathcal{O}_{M}$ is a principal ideal. Let $g$ be a local generator of $\mathcal{I}$ in a neighbourhood $U$ of $a \in M$ with coordinates $\left(x_{1}, \ldots, x_{m}\right)$. Let $d=\operatorname{ord}_{a} \mathcal{I}$. (By making a linear coordinate change), we can assume that $\partial^{d} g / \partial x_{m}^{d}$ is nonvanishing. Let $z:=\partial^{d-1} g / \partial x_{n}^{d-1}$. Then $N:=V(z)$ is a hypersurface of maximal contact for $\left.\underline{\mathcal{I}}\right|_{U}:=\left(U, U, \emptyset,\left.\mathcal{I}\right|_{U}, d\right)$, and $\left.\underline{\mathcal{I}}\right|_{U}$ is equivalent to $\underline{\mathcal{C}}_{x_{n}, N}(\underline{\mathcal{I}}):=$ $\left(U, N, \emptyset,\left.\mathcal{C}_{x_{n}}^{d-1}(\mathcal{I})\right|_{N}, d_{\underline{\mathcal{C}}_{x_{n}, N}(\underline{\mathcal{I}})}\right)$, where $\mathcal{C}_{x_{n}}^{d-1}(\mathcal{I}):=\sum_{k=0}^{d-1}\left(\left(\partial^{k} g / \partial x_{n}^{k}\right), d-k\right)$. 


\section{$\S 5 . \quad$ Proof of Resolution of Singularities of a Marked Ideal}

In this section, we prove Theorem 1.3. Let $\underline{\mathcal{I}}=(M, N, E, \mathcal{I}, d)$ denote a marked ideal, where $d>0$. The proof is by induction on $\operatorname{dim} \underline{\mathcal{I}}:=\operatorname{dim} N$.

First suppose $\operatorname{dim} N=0$. If $\mathcal{I}=0$, then $\operatorname{cosupp} \underline{\mathcal{I}}=N$ and we can blow up with centre $N$ to resolve the singularities of $\underline{\mathcal{I}}$. If $\mathcal{I} \neq 0$, then $\operatorname{cosupp} \underline{\mathcal{I}}=\emptyset$ (since $d>0$ ), so that $\underline{\mathcal{I}}$ is already resolved.

The inductive step breaks up into the two independent steps I and II formulated in Section 1.

Step I. Maximal order case. Let $\underline{\mathcal{I}}=(M, N, E, \mathcal{I}, d)$ be a marked ideal of maximal order.

Case A. $E=\emptyset$. Let $a \in \operatorname{cosupp} \underline{\mathcal{I}}$. By Corollary 4.1, there is a hypersurface of maximal contact $P$ for $\underline{\mathcal{I}}$ at $a ; P \subset N \cap U$, where $U$ is a neighbourhood of $a$ in $M$. Define

$$
\underline{\mathcal{C}}_{U}(\underline{\mathcal{I}}):=\underline{\mathcal{C}}_{\emptyset, P}(\underline{\mathcal{I}})=\left(U, P, \emptyset,\left.\underline{\mathcal{C}}_{\emptyset}^{d-1}(\underline{\mathcal{I}})\right|_{P}, d_{\underline{\mathcal{C}}}\right),
$$

where $d_{\underline{\mathcal{C}}}=d_{\underline{\mathcal{C}}_{\theta}^{d-1}(\underline{\mathcal{I}})}$. Then $\underline{\mathcal{C}}_{U}(\underline{\mathcal{I}})$ is equivalent to $\left.\underline{\mathcal{I}}\right|_{U}=\left(U, N \cap U, \emptyset,\left.\mathcal{I}\right|_{N \cap U}, d\right)$, according to Corollary 4.2. Therefore, a resolution of singularities of $\underline{\mathcal{C}}_{U}(\underline{\mathcal{I}})$ (which exists by induction) is a resolution of singularities of $\left.\underline{\mathcal{I}}\right|_{U}$.

The marked ideals $\underline{\mathcal{C}}_{U}(\underline{\mathcal{I}})$ and $\underline{\mathcal{C}}_{V}(\underline{\mathcal{I}})$ defined in two overlapping charts $U$ and $V$ are equivalent in $U \cap V$ (since both are equivalent to $\left.\underline{\mathcal{I}}\right|_{U \cap V}$ ); therefore, by functoriality in dimension $n-1$, their resolution sequences are the same over $U \cap V$ (not counting blowings-up that restrict to isomorphisms over $U \cap V$ ).

Claim 5.1. It follows that the resolution sequences for the $\underline{\mathcal{C}}_{U}(\underline{\mathcal{I}})($ defined locally) patch together to give functorial resolution of singularities of a marked ideal $\underline{\mathcal{I}}$.

The problem here is that, to glue together the local centres of blowing up, we have to know which nontrivial centres in the various charts $U$ should be taken first, etc. We leave this claim to Section 7 below, where we give two different proofs.

Case B. General maximal order case. Let $\underline{I}_{\emptyset}$ denote the marked ideal $(M, N, \emptyset, \mathcal{I}, d)$. Let $a \in \operatorname{cosupp} \underline{\mathcal{I}}=\operatorname{cosupp} \underline{\mathcal{I}}_{\emptyset}$. By Corollary 4.1, there exists an (irreducible) hypersurface of maximal contact $P$ for $\underline{I}_{\emptyset}$, defined in a neighbourhood $U$ of $a$. We introduce $\underline{\mathcal{C}}:=\underline{\mathcal{C}}_{U}\left(\underline{\mathcal{I}}_{\emptyset}\right)=\left(U, P, \emptyset, \mathcal{C}, d_{\underline{\mathcal{C}}}\right)$, as in Case A above.

If $x \in N$, set

$$
s(x):=\#\{H \in E: x \in H\}
$$


(where " $H \in E$ " means " $H$ is a component of $E$ ", and \# denotes the cardinality of a finite set). After any sequence of test transformations of $\underline{\mathcal{I}}$, we will continue to write $E$ (as opposed to $E_{t}$ ) for the divisor whose components are the strict transforms of those of $E$, with the same ordering as in $E$, and we will continue to write $s(x)$ for $\#\{H \in E: x \in H\}$.

Let $s:=\max \{s(x): x \in \operatorname{cosupp} \underline{\mathcal{I}}\}$, and let $\operatorname{Sub}(E, s)$ denote the set of $s$-element subsets of $E$ (i.e., of the set of components of $E$ ). Let $\underline{\mathcal{E}}$ denote the marked ideal $\underline{\mathcal{E}}:=\left(U, P, \emptyset, \mathcal{E}, d_{\mathcal{C}}\right)$, where

$$
\mathcal{E}:=\prod_{\Lambda \in \operatorname{Sub}(E, s)} \sum_{H \in \Lambda} \mathcal{I}_{H}^{d_{\mathcal{C}}} \cdot \mathcal{O}_{P}
$$

Set $\underline{\mathcal{J}}:=\underline{\mathcal{C}}+\underline{\mathcal{E}}$.

Clearly, $\operatorname{cosupp} \underline{\mathcal{E}}=\{x \in P: s(x) \geq s\}$, so that

$$
\operatorname{cosupp} \underline{\mathcal{J}}=\left.\operatorname{cosupp} \underline{\mathcal{I}}\right|_{U} \bigcap\{x \in P: s(x)=s\},
$$

(and likewise after any sequence of test transformations of $\underline{\mathcal{J}}$ ). Thus any sequence of test transformations of $\mathcal{J}$ is a sequence of test transformations of $\left.\underline{I}\right|_{U}$ (and the centre $C$ of each admissible blowing-up lies inside the intersection of the components of $E$ that pass through a point of $C$ ). Therefore, the equivalence class of $\underline{\mathcal{J}}$ depends only on that of $\left.\underline{\mathcal{I}}\right|_{U}$, and blowings-up which are admissible for $\underline{\mathcal{I}}$ are also admissible for $\left.\underline{\mathcal{I}}\right|_{U}$.

By induction on dimension, there is a resolution of singularities of $\underline{\mathcal{J}}$. We can define $\underline{\mathcal{J}}$ for each element of a finite covering $\{U\}$ of $M$ and, as in Claim 5.1 , the resolution sequences for the $\underline{\mathcal{J}}$ patch together to define a sequence of admissible blowings-up of $\underline{\mathcal{I}}$. (See Section 7.)

If this sequence of blowings-up resolves $\underline{\mathcal{I}}$, then we have finished Case B. Otherwise, $\operatorname{cosupp} \underline{\mathcal{I}}_{j}$ and $\{x: s(x)=s\}$ become disjoint for some index $j$ of the blowings-up sequence, say for $j=q_{1}$ (i.e., $s(x)<s$, for all $x \in \operatorname{cosupp} \underline{\mathcal{I}}_{j}$ ). In this case, we repeat the process using $\underline{\mathcal{C}}_{q_{1}}:=\underline{\mathcal{C}}_{U}\left(\underline{\mathcal{I}}_{\emptyset}\right)_{q_{1}}$ in place of $\underline{\mathcal{C}}$, and using the new value $s_{1}$ of $\max \left\{s(x): x \in \operatorname{cosupp} \underline{\mathcal{I}}_{q_{1}}\right\}$ in place of $s$. (Here $s(x)$ has the same meaning as above - it is defined using $E$ as opposed to $E_{q_{1}}$. The process above is repeated using $\underline{\mathcal{J}}_{1}:=\underline{\mathcal{C}}_{q_{1}}+\underline{\mathcal{E}}_{1}$ in place of $\underline{\mathcal{J}}$, where $\underline{\mathcal{E}}_{1}$ is given by (5.2) with $s_{1}$ in place of $s$.)

We thus get marked ideals $\underline{\mathcal{I}}_{q_{1}}, \ldots, \underline{\mathcal{I}}_{q_{k}}, \ldots$ with $s>s_{1}>\cdots>s_{k}>\cdots$, and, for each $k$ and $U$, a marked ideal $\underline{\mathcal{J}}_{k}=\underline{\mathcal{C}}_{q_{k}}+\underline{\mathcal{E}}_{k}$ analogous to $\underline{\mathcal{J}}_{1}$. After finitely many steps as above, $\underline{\mathcal{I}}$ is resolved. (If, after $k$ steps, $\underline{\mathcal{I}}$ is not resolved but $s_{k}=0$, then $\underline{\mathcal{I}}$ is resolved by the next step.) So we have completed Case $B$ and therefore Step I. 
Step II. General case. Let $\underline{\mathcal{I}}=(M, N, E, \mathcal{I}, d)$ be an arbitrary marked ideal.

First suppose that $\mathcal{I}=0$ (so that $\operatorname{cosupp} \underline{\mathcal{I}}=N)$. Then we can blow up with centre $N$ to resolve singularities.

Now suppose that $\mathcal{I} \neq 0$. Write

$$
\mathcal{I}=\mathcal{M}(\underline{\mathcal{I}}) \cdot \mathcal{N}(\underline{\mathcal{I}})
$$

where $\mathcal{M}(\underline{\mathcal{I}})$ is a product of prime ideals defining irreducible components of the elements of $E$, and $\mathcal{N}(\underline{\mathcal{I}})$ is divisible by no such prime ideal. We consider two cases.

Case A. Monomial case $\mathcal{I}=\mathcal{M}(\underline{\mathcal{I}})$. For any sequence of admissible blowings-up $\sigma_{j}$ of $\underline{\mathcal{I}}$, let us order the collection of subsets of each $E_{j}$ as follows: Suppose that $E=\left\{H_{1}, \ldots, H_{q}\right\}$. Write $E_{0}:=E=\left\{H_{1}, \ldots, H_{q}\right\}=$ : $\left\{H_{1}^{0}, \ldots, H_{q}^{0}\right\}$. For each $j=1, \ldots$, write $E_{j}=\left\{H_{1}^{j}, \ldots, H_{q+j}^{j}\right\}$, where $H_{i}^{j}$ denotes the strict transform of $H_{i}^{j-1}$ by $\sigma_{j}$, if $i<q+j$, and $H_{q+j}^{j}=\sigma_{j}^{-1}\left(C_{j-1}\right)$. Now, we associate to each subset $I$ of $E_{j}$ the finite sequence $\left(\delta_{1}, \ldots, \delta_{q+j}\right)$, where $\delta_{i}=0$ if $H_{i}^{j} \notin I$, and $\delta_{i}=1$ if $H_{i}^{j} \in I$. Then we order the subsets $I$ of $E_{j}$, for all $j$, by the lexicographic ordering of such sequences.

Let $a \in \operatorname{cosupp} \underline{\mathcal{I}}$. In a neighbourhood of $a$, we can write $\mathcal{I}=\mathcal{I}_{H_{i_{1}}}^{\alpha_{1}} \cdots \mathcal{I}_{H_{i_{r}}}^{\alpha_{r}}$ (where $a \in H_{i_{1}} \cap \cdots \cap H_{i_{r}}$ and where we write $\mathcal{I}_{H_{i_{k}}}$ instead of $\mathcal{I}_{H_{i_{k}}} \cdot \mathcal{O}_{N}$ to simplify the notation); in particular, $\alpha_{1}+\cdots+\alpha_{r}>d$. (In Theorem 6.1 below, we will see that $\mu_{a}(\underline{\mathcal{I}})=\left(\alpha_{1}+\cdots+\alpha_{r}\right) / d$ is a local invariant of the equivalence class of $\underline{\mathcal{I}}$.) Then (in the neighbourhood above) $\operatorname{cosupp} \underline{\mathcal{I}}=\cup Z_{I}$, where each $Z_{I}:=N \cap \bigcap_{H \in I} H$, and $I$ runs over the smallest subsets of $\left\{H_{i_{1}}, \ldots, H_{i_{r}}\right\}$ such that $\sum_{l \in I} \alpha_{l} \geq d$; in other words, $I$ runs over the subsets of $\left\{H_{i_{1}}, \ldots, H_{i_{r}}\right\}$ such that

$$
0 \leq \sum_{l \in I} \alpha_{l}-d<\alpha_{k}, \quad \text { for all } k \in I .
$$

(We have simplified the notation by identifying subsets of $\left\{H_{i_{1}}, \ldots, H_{i_{r}}\right\}$ with subsets of $\{1, \ldots, r\}$.)

Let $J(a)$ denote the maximum of these subsets $I$ (with respect to the order above). Clearly, $J(x)$ is Zariski upper-semicontinuous on $\operatorname{cosupp} \underline{\mathcal{I}}$, and the maximum locus of $J(x)$ consists of at most one irreducible component of cosupp $\underline{\mathcal{I}}$ through each point.

Consider the blowing-up $\sigma$ with centre $C$ given by the maximum locus of $J(\cdot)$. If $a \in C$, then (in a neighbourhood as above), $C=N \cap \bigcap_{l \in J(a)} H_{i_{l}}$. We can choose local coordinates $\left(x_{1}, \ldots, x_{n}\right)$ for $N$ at $a$ such that, for each $k \in J(a)$, $x_{k}$ is a local generator $x_{H_{i_{k}}}$ of the ideal $\left.\mathcal{I}_{H_{i_{k}}}\right|_{N}$. Then, in the $x_{k}$-chart of the 
blowing-up $\sigma$, the transform of $\mathcal{I}$ is given by

$$
\mathcal{I}^{\prime}=\mathcal{I}_{H_{i_{1}}^{\prime}}^{\alpha_{1}} \cdots \mathcal{I}_{\sigma^{-1}(C)}^{\sum_{J(a)} \alpha_{l}-d} \cdots \mathcal{I}_{H_{i_{r}}^{\prime}}^{\alpha_{r}},
$$

(with $\mathcal{I}_{\sigma^{-1}(C)}^{\sum_{J(a)} \alpha_{l}-d}$ in the $k$ 'th place). Since $\sum_{l} \alpha_{l}-d<\alpha_{k}, \mu_{a^{\prime}}\left(\underline{\mathcal{I}}^{\prime}\right)=\mu_{a}(\underline{\mathcal{I}})-$ $p / d$, where $p$ is a positive integer, over all points $a$ of some component of $\operatorname{cosupp} \underline{\mathcal{I}}$. We therefore resolve singularities after a finite number of steps.

Case B. General case. Set

$$
\operatorname{ord} \mathcal{N}(\underline{\mathcal{I}}):=\max _{x \in \operatorname{cosupp} \underline{\underline{\mathcal{I}}}} \operatorname{ord}_{x} \mathcal{N}(\underline{\mathcal{I}}),
$$

and define marked ideals

$$
\begin{aligned}
& \underline{\mathcal{N}}(\underline{\mathcal{I}}):=(M, N, E, \mathcal{N}(\underline{\mathcal{I}}), \operatorname{ord} \mathcal{N}(\underline{\mathcal{I}})), \\
& \underline{\mathcal{M}}(\underline{\mathcal{I}}):=(M, N, E, \mathcal{M}(\underline{\mathcal{I}}), d-\operatorname{ord} \mathcal{N}(\underline{\mathcal{I}})) .
\end{aligned}
$$

We define the companion ideal of $\underline{\mathcal{I}}=(\mathcal{I}, d)$ as

$$
\underline{\mathcal{G}}(\underline{\mathcal{I}}):= \begin{cases}\underline{\mathcal{N}}(\underline{\mathcal{I}})+\underline{\mathcal{M}}(\underline{\mathcal{I}}), & \text { if } \operatorname{ord} \mathcal{N}(\underline{\mathcal{I}})<d ; \\ \underline{\mathcal{N}}(\underline{\mathcal{I}}), & \text { if } \operatorname{ord} \mathcal{N}(\underline{\mathcal{I}}) \geq d .\end{cases}
$$

(In the following, we treat both cases simultaneously by defining the cosupport and transforms of $\underline{\mathcal{M}}(\underline{\mathcal{I}})$ in the case that $\operatorname{ord} \mathcal{N}(\underline{\mathcal{I}}) \geq d$ exactly as in Section 2, even though $d-\operatorname{ord} \mathcal{N}(\underline{\mathcal{I}}) \leq 0$; e.g., $\operatorname{cosupp} \underline{\mathcal{M}}(\underline{\mathcal{I}})=N$ in this case.) Then $\underline{\mathcal{G}}(\underline{\mathcal{I}})$ is of maximal order (by Lemma 3.8(4)) and, for any sequence of test transformations of $\underline{\mathcal{G}}(\underline{\mathcal{I}})$,

$$
\begin{aligned}
\operatorname{cosupp} \underline{\mathcal{G}}(\underline{\mathcal{I}})_{j} & =\operatorname{cosupp} \underline{\mathcal{N}}(\underline{\mathcal{I}})_{j} \bigcap \operatorname{cosupp} \underline{\mathcal{M}}(\underline{\mathcal{I}})_{j} \\
& \subset \operatorname{cosupp} \underline{\mathcal{N}}(\underline{\mathcal{I}})_{j} \bigcap \operatorname{cosupp} \underline{\mathcal{I}}_{j} .
\end{aligned}
$$

By Lemma $3.7, \underline{\mathcal{I}}_{j}=\underline{\mathcal{M}}(\underline{\mathcal{I}})_{j} \cdot \underline{\mathcal{N}}(\underline{\mathcal{I}})_{j}$.

Remark 5.2. For any sequence of admissible blowings-up of $\underline{\mathcal{G}}(\underline{\mathcal{I}})$, it is clear that $\underline{\mathcal{N}}(\underline{\mathcal{I}})_{j}=\underline{\mathcal{N}}\left(\underline{\mathcal{I}}_{j}\right), \underline{\mathcal{M}}(\underline{\mathcal{I}})_{j}=\underline{\mathcal{M}}\left(\underline{\mathcal{I}}_{j}\right)$, and cosupp $\underline{\mathcal{G}}(\underline{\mathcal{I}})_{j}=$ cosupp $\underline{\mathcal{N}}(\underline{\mathcal{I}})_{j} \cap \operatorname{cosupp} \underline{\mathcal{I}}_{j}$.

In Theorems 6.1 and 6.2 below, we will prove that, if $a \in \operatorname{cosupp} \underline{\mathcal{I}}$, then

$$
\mu_{a}(\underline{\mathcal{I}}):=\frac{\operatorname{ord}_{a} \mathcal{I}}{d} \quad \text { and } \quad \mu_{H, a}(\underline{\mathcal{I}}):=\frac{\operatorname{ord}_{H, a} \mathcal{I}}{d}, H \in E,
$$

depend only on the equivalence class of $\underline{\mathcal{I}}$ and $\operatorname{dim} N .\left(\operatorname{ord}_{H, a} \mathcal{I}\right.$ denotes the order of $\mathcal{I} \subset \mathcal{O}_{N}$ along $\left.H\right|_{N}$ at $a$; i.e., the largest $\mu \in \mathbb{N}$ such that $\mathcal{I}_{a} \subset \mathcal{I}_{\left.H\right|_{N}, a}^{\mu}$.) The following is a corollary of these results. 
Corollary 5.3. $\quad$ The equivalence class of $\underline{\mathcal{G}}(\underline{\mathcal{I}})$ depends only on the equivalence class of $\underline{\mathcal{I}}$ and $\operatorname{dim} N$.

Proof. The divisor (with rational coefficients) $\mathcal{M}(\underline{\mathcal{I}})^{1 / d}$ depends only on the equivalence class of $\underline{\mathcal{I}}$, by Theorems 6.1 and 6.2. Over any sequence of test transformations of $\underline{\mathcal{G}}(\underline{\mathcal{I}})$,

$$
\begin{aligned}
\operatorname{cosupp} \underline{\mathcal{G}}(\underline{\mathcal{I}})_{j}=\left\{x \in \operatorname{cosupp} \underline{\mathcal{I}}_{j}: \quad\right. & \operatorname{ord}_{x} \mathcal{N}(\underline{\mathcal{I}})_{j} \geq \operatorname{ord} \mathcal{N}(\underline{\mathcal{I}}) \\
& \left.\operatorname{ord}_{x} \mathcal{M}(\underline{\mathcal{I}})_{j} \geq d-\operatorname{ord} \mathcal{N}(\underline{\mathcal{I}})\right\} \\
=\left\{x \in \operatorname{cosupp} \underline{\mathcal{I}}_{j}:\right. & \mu_{x}\left(\underline{\mathcal{I}}_{j}\right)-\frac{1}{d} \operatorname{ord}_{x} \mathcal{M}(\underline{\mathcal{I}})_{j} \geq \frac{1}{d} \operatorname{ord} \mathcal{N}(\underline{\mathcal{I}}), \\
& \left.\frac{1}{d} \operatorname{ord}_{x} \mathcal{M}(\underline{\mathcal{I}})_{j} \geq 1-\frac{1}{d} \operatorname{ord} \mathcal{N}(\underline{\mathcal{I}})\right\} .
\end{aligned}
$$

All terms in the latter condition depend only on the equivalence class of $\underline{\mathcal{I}}$ and $\operatorname{dim} N$.

Now, we can resolve the singularities of the marked ideal of maximal order $\underline{\mathcal{G}}(\underline{\mathcal{I}})$, using Step I. The blowings-up involved are admissible for $\underline{\mathcal{I}}$. Such a resolution leads, after a finite number of steps (say $r_{1}$ steps) to a marked ideal $\underline{\mathcal{I}}_{r_{1}}$ such that either ord $\mathcal{N}\left(\underline{\mathcal{I}}_{r_{1}}\right)<\operatorname{ord} \mathcal{N}(\underline{\mathcal{I}})$, or $\operatorname{cosupp} \underline{\mathcal{I}}_{r_{1}}=\emptyset$. (See Remark 5.2.) In the latter case, we have resolved the singularities of $\underline{\mathcal{I}}$. In the former case, we can repeat the process for $\underline{\mathcal{I}}_{r_{1}}=\left(\mathcal{I}_{r_{1}}, d\right), \ldots$. We get marked ideals $\underline{\underline{I}}_{r_{1}}, \ldots, \underline{\mathcal{I}}_{r_{k}}, \ldots$ such that

$$
\operatorname{ord} \mathcal{N}(\underline{\mathcal{I}})>\operatorname{ord} \mathcal{N}\left(\underline{\mathcal{I}}_{r_{1}}\right)>\cdots>\operatorname{ord} \mathcal{N}\left(\underline{\mathcal{I}}_{r_{k}}\right)>\cdots .
$$

The process terminates after a finite number of steps, when either we have $\operatorname{ord} \mathcal{N}\left(\underline{\mathcal{I}}_{r_{k}}\right)=0$ (i.e., we have reduced to the monomial case $\underline{\underline{I}}_{r_{k}}=\underline{\mathcal{M}}\left(\underline{\mathcal{I}}_{r_{k}}\right)$ ), or we have cosupp $\underline{\mathcal{I}}_{r_{k}}=\emptyset$ (i.e., we have resolved singularities).

This will complete the proof of functorial resolution of singularities of a marked ideal (Theorem 1.3) once we establish Claim 5.1 and prove Theorems $6.1,6.2$.

\section{$\S 6 . \quad$ Invariants of a Marked Ideal}

If $\underline{\mathcal{I}}=(M, N, E, \mathcal{I}, d)$ is a marked ideal and $a \in \operatorname{cosupp} \underline{\mathcal{I}}$, we define $\mu_{a}(\underline{\mathcal{I}}):=\operatorname{ord}_{a} \mathcal{I} / d$ and $\mu_{H, a}(\underline{\mathcal{I}}):=\operatorname{ord}_{H, a} \mathcal{I} / d, H \in E$, as in (5.3). These orders can be calculated in local coordinates. To prove the two theorems below, we find sequences of test transformations depending only on the equivalence class 
of $\underline{\mathcal{I}}$, such that $\mu_{a}(\underline{\mathcal{I}})$ and the $\mu_{H, a}(\underline{\mathcal{I}})$ can be calculated also from orders of vanishing of the transformed ideals along a "horizontal divisor" introduced by product with a line. The first theorem is due to Hironaka [Hi2].

Theorem 6.1. Let $\underline{\mathcal{I}}=(M, N, E, \mathcal{I}, d)$ and $\underline{\mathcal{J}}=(M, P, E, \mathcal{J}, e)$ be marked ideals. Suppose that $\underline{\mathcal{I}}$ and $\underline{\mathcal{I}}$ are weak-equivalent (Definitions 2.4). Let $a \in \operatorname{cosupp} \underline{\mathcal{I}}=\operatorname{cosupp} \underline{\mathcal{I}}$. If $\operatorname{dim} N=\operatorname{dim} P$, then $\mu_{a}(\underline{\mathcal{I}})=\mu_{a}(\underline{\mathcal{J}})$. If $\operatorname{dim} N>\operatorname{dim} P$, then $\mu_{a}(\underline{\mathcal{I}})=1$.

Proof. We have $\mu_{a}(\underline{\mathcal{I}})=\infty$ if and only if $\mathcal{I}=0$ at $a$, i.e., cosupp $\underline{\mathcal{I}}$ is smooth of dimension $=\operatorname{dim} N$ at $a$.

Suppose that $\mu_{a}(\underline{\mathcal{I}})<\infty$. Set $\underline{\mathcal{I}}_{0}:=\underline{\mathcal{I}}, \underline{\mathcal{I}}_{0}=\left(M_{0}, N_{0}, E_{0}, \mathcal{I}_{0}, d\right)$. Let $\sigma_{1}$ : $M_{1}:=M_{0} \times \mathbb{A}^{1} \rightarrow M_{0}$ denote the projection, and let $\underline{\mathcal{I}}_{1}=\left(M_{1}, N_{1}, E_{1}, \mathcal{I}_{1}, d\right)$ denote the transform of $\underline{\mathcal{I}}_{0}$ by $\sigma_{1}$ (Defns. 2.4). Let $a_{1}:=(a, 0)$ and let $\Gamma_{1}:=$ $\{a\} \times \mathbb{A}^{1} \subset \operatorname{cosupp} \underline{\mathcal{I}}_{1}=\sigma_{1}^{-1}\left(\operatorname{cosupp} \underline{\mathcal{I}}_{0}\right)$. Consider a sequence of admissible blowings-up

$$
M_{1} \stackrel{\sigma_{2}}{\longleftarrow} M_{2} \longleftarrow \cdots \longleftarrow M_{j} \stackrel{\sigma_{j+1}}{\longleftarrow} M_{j+1} \longleftarrow \cdots
$$

and the corresponding transforms $\underline{\underline{I}}_{j}=\left(M_{j}, N_{j}, E_{j}, \mathcal{I}_{j}, d\right)$ of $\underline{\mathcal{I}}_{1}$, where, for each $j \geq 1, \sigma_{j+1}$ denotes the blowing-up with center $a_{j}$, and the $a_{j}$ are defined inductively as follows: For each $j \geq 1, a_{j+1}:=\Gamma_{j+1} \cap D_{j+1}$, where $D_{j+1}$ is the exceptional divisor $\sigma_{j+1}^{-1}\left(a_{j}\right)$ and $\Gamma_{j+1}$ is the strict transform of $\Gamma_{j}$.

Clearly, each $\Gamma_{j+1} \subset \operatorname{cosupp} \underline{\mathcal{I}}_{j+1}$, since $\Gamma_{j+1} \backslash\left\{a_{j+1}\right\} \subset \operatorname{cosupp} \underline{\mathcal{I}}_{j+1}$.

We introduce a subset $S$ of $\mathbb{N} \times \mathbb{N}$ depending only on $\operatorname{dim} N$ and the weakequivalence class of $\underline{\mathcal{I}}$, as follows: Let $r=\operatorname{dim} M-\operatorname{dim} N$. Note that, for each $j \geq 1, D_{j+1} \cap N_{j+1}$ is smooth, and has codimension $r$ in $D_{j+1}$ and codimension 1 in $N_{j+1}$. We say that $(j, 0) \in S, j \geq 1$, if, after $j$ blowings-up $\sigma_{2}, \ldots, \sigma_{j+1}$ as above, $D_{j+1} \cap \operatorname{cosupp} \underline{\mathcal{I}}_{j+1}$ contains (i.e., is) a smooth subvariety of codimension $r$ in $D_{j+1}$ at $a_{j+1}$.

In this case, $D_{j+1} \cap \operatorname{cosupp} \underline{\mathcal{I}}_{j+1}=D_{j+1} \cap N_{j+1}$ at $a_{j+1}$, and the condition $D_{j+1} \cap N_{j+1} \subset \operatorname{cosupp} \underline{\mathcal{I}}_{j+1}$ means that $\operatorname{ord}_{D_{j+1}, a_{j+1}} \underline{\mathcal{I}}_{j+1} \geq d$. The latter inequality is equivalent to $j\left(\mu_{a}(\underline{\mathcal{I}})-1\right) d \geq d$, i.e., $j\left(\mu_{a}(\underline{\mathcal{I}})-1\right) \geq 1$. This can be proved by induction on $j$, by a simple formal Taylor series calculation: For each $j, \mathcal{I}_{j+1, a_{j+1}}=\mathcal{I}_{D_{j+1}, a_{j+1}}^{-j d} \cdot\left(\mathcal{I}_{a} \cdot \mathcal{O}_{N_{j+1}, a_{j+1}}\right)$. But ord $a_{j+1} \underline{\mathcal{I}}_{j+1}=\operatorname{ord}_{a_{j}} \underline{\mathcal{I}}_{j}=$ $\cdots=\operatorname{ord}_{a} \underline{\mathcal{I}}$; therefore, $\mathcal{I}_{a} \cdot \mathcal{O}_{N_{j+1}, a_{j+1}}$ is divisible by $\mathcal{I}_{D_{j+1}, a_{j+1}}^{\text {jord } \mathcal{I}}$ and not by any higher power. Since $\operatorname{ord}_{a} \mathcal{I}=\mu_{a}(\underline{\mathcal{I}}) d$, we have $\mathcal{I}_{j+1, a_{j+1}}=\mathcal{I}_{D_{j+1}, a_{j+1}}^{j\left(\mu^{(\mathcal{I})}-1\right) d} \cdot \tilde{\mathcal{I}}_{a_{j+1}}$, where $\tilde{\mathcal{I}}_{a_{j+1}}$ is not divisible by $\mathcal{I}_{D_{j+1}, a_{j+1}}$.

In particular, since $\mu_{a}(\underline{\mathcal{I}}) \geq 1,(j, 0) \notin S$ for all $j \geq 1$ if and only if $\mu_{a}(\underline{\mathcal{I}})=1$. 
It follows that if $\underline{\mathcal{I}}$ is weak-equivalent to $\underline{\mathcal{J}}$, where $\operatorname{dim} P<\operatorname{dim} N$, then $\mu_{a}(\underline{\mathcal{I}})=1$, since $D_{j+1} \cap \operatorname{cosupp} \underline{\mathcal{I}}_{j+1}=D_{j+1} \cap \operatorname{cosupp} \underline{\mathcal{J}}_{j+1} \subset D_{j+1} \cap P_{j+1}$ cannot contain a smooth subvariety of codimension $r$ in $D_{j+1}$.

Now suppose that $(j, 0) \in S$, for some $j \geq 1$. Then we can consider the blowing-up $\sigma_{j+2}: M_{j+2} \rightarrow M_{j+1}$ with centre $C_{j+1}=D_{j+1} \cap N_{j+1}$. Note that $\left.\sigma_{j+2}\right|_{N_{j+2}}: N_{j+2} \rightarrow N_{j+1}$ is the identity! Set $G_{j+2}:=$ strict transform of $G_{j+1}$ and $a_{j+2}:=G_{j+2} \cap D_{j+2}$. We say that $(j, 1) \in S$ if $D_{j+2} \cap \operatorname{cosupp} \underline{\mathcal{I}}_{j+2}$ contains a smooth subvariety of codimension $r$ in $D_{j+2}$ at $a_{j+2}$.

If so, then again $D_{j+2} \cap \operatorname{cosupp} \underline{\mathcal{I}}_{j+2}=D_{j+2} \cap N_{j+2}$. Since $\mathcal{I}_{j+1}=$ $\mathcal{I}_{D_{j+1}}^{j\left(\mu_{a}(\mathcal{I})-1\right) d} \cdot \tilde{\mathcal{I}}_{j+1}$ at $a_{j+1}$, where $\tilde{\mathcal{I}}_{j+1}$ is not divisible by $\mathcal{I}_{D_{j+1}}$, we see that $\mathcal{I}_{j+2}=\mathcal{I}_{D_{j+2}}^{j\left(\mu_{a}(\underline{\mathcal{I}})-1\right) d-d} \cdot \tilde{\mathcal{I}}_{j+2}$ at $a_{j+2}$, where $\tilde{\mathcal{I}}_{j+2}=\tilde{\mathcal{I}}_{j+1}$ is not divisible by $\mathcal{I}_{D_{j+2}}$. Therefore, $(j, 1) \in S$ if and only if $j\left(\mu_{a}(\underline{\mathcal{I}})-1\right) d-d \geq d$.

We continue inductively: If $i \geq 1$ and $(j, i-1) \in S$, let $\sigma_{j+i+1}: M_{j+i+1} \rightarrow$ $M_{j+i}$ denote the blowing-up with centre $C_{j+i}=D_{j+i} \cap N_{j+i}$. Set $G_{j+i+1}:=$ strict transform of $G_{j+i}$ and $a_{j+i+1}:=G_{j+i+1} \cap D_{j+i+1}$. We say that $(j, i) \in S$ if $D_{j+i+1} \cap \operatorname{cosupp} \underline{\mathcal{I}}_{j+i+1}$ is smooth and of codimension $r$ in $D_{j+i+1}$; i.e., $(j, i) \in S$ if and only if $j\left(\mu_{a}(\underline{\mathcal{I}})-1\right)-i \geq 1$.

According to its definition, $S$ depends only on $\operatorname{dim} N$ and the weakequivalence class of $\underline{\mathcal{I}}$ (and the point $a$ ). We have shown that $S=\emptyset$ if and only if $\mu_{a}(\underline{\mathcal{I}})=1$, and, if $S \neq \emptyset$, then

$$
S=\left\{(j, i) \in \mathbb{N} \times \mathbb{N}: j\left(\mu_{a}(\underline{\mathcal{I}})-1\right)-i \geq 1\right\}
$$

therefore, $\mu_{a}(\underline{\mathcal{I}})$ is uniquely determined by $S . \quad\left(\mu_{a}(\underline{\mathcal{I}})=1+\sup _{(j, i) \in S}(i+\right.$ $1) / j$.)

Theorem 6.2. Let $\underline{\mathcal{I}}=(M, N, E, \mathcal{I}, d)$ be a marked ideal and let a $\in$ cosupp $\underline{\mathcal{I}}$. Consider $H \in E$. Then $\mu_{H, a}(\underline{\mathcal{I}})$ depends only on the equivalence class of $\underline{\mathcal{I}}$ and the dimension of $N$ at a. (See Defns.2.5.)

Proof. Suppose that $H \ni a$. Set $\underline{\mathcal{I}}_{0}:=\underline{\mathcal{I}}, \underline{\mathcal{I}}_{0}=\left(M_{0}, N_{0}, E_{0}, \mathcal{I}_{0}, d\right)$. We can choose local coordinates $\left(x_{1}, \ldots, x_{n}\right)$ for $N$ at $a$, such that $\left.H\right|_{N_{0}}=\left\{x_{1}=\right.$ $0\}$. Let $\sigma_{1}: M_{1}=M_{0} \times \mathbb{A}^{1} \rightarrow M_{0}$ denote the projection, let $a_{1}=(a, 0)$ and let $\underline{\mathcal{I}}_{1}=\left(M_{1}, N_{1}, E_{1}, \mathcal{I}_{1}, d\right)$ denote the transform of $\underline{\mathcal{I}}_{0}$ by $\sigma_{1}$. (Thus $\left(x_{1}, \ldots, x_{n}, t\right)$ are local coordinates for $N_{1}$ at $a_{1}$.) Let $H_{0}^{1}:=M_{0} \times\{0\}$ (so that $\left.\left.H_{0}^{1}\right|_{N_{1}}=\{t=0\}\right)$ and let $H_{1}^{1}:=\sigma^{-1}(H)\left(\left(\right.\right.$ so that $\left.H_{1}^{1}\right|_{N_{1}}=\left\{x_{1}=0\right\}$.) Set $\Gamma_{1}:=\{a\} \times \mathbb{A}^{1}$.

We follow $\sigma_{1}$ by a sequence of exceptional blowings-up

$$
M_{1} \stackrel{\sigma_{2}}{\longleftarrow} M_{2} \longleftarrow \cdots \longleftarrow M_{j} \stackrel{\sigma_{j+1}}{\longleftarrow} M_{j+1} \longleftarrow \cdots,
$$


where (for all $j \geq 1$ ) we inductively define $\sigma_{j+1}$ as the blowing-up with centre $C_{j}:=H_{0}^{j} \cap H_{1}^{j}$, and set $H_{0}^{j+1}:=\sigma_{j+1}^{-1}\left(C_{j}\right), H_{1}^{j+1}:=$ strict transform of $H_{1}^{j}$ by $\sigma_{j+1}, \Gamma_{j+1}:=$ strict transform of $\Gamma_{j}, a_{j+1}:=\Gamma_{j+1} \cap H_{0}^{j+1}=C_{j+1} \cap \sigma_{j+1}^{-1}\left(a_{j}\right)$. Then, for each $j \geq 1, N_{j+1}$ has a chart with coordinates $(x, t)=\left(x_{1}, \ldots, x_{n}, t\right)$ at $a_{j+1}$ in which $\left(\sigma_{2} \circ \cdots \circ \sigma_{j+1}\right)(x, t)=\left(t^{j} x_{1}, x_{2}, \ldots, x_{n}, t\right)$.

Write $\mathcal{I}=\mathcal{I}_{H}^{\mu_{H, a}(\underline{\mathcal{I}}) d} \cdot \mathcal{J}$ at $a$, where $\mathcal{J}$ is not divisible by $\mathcal{I}_{H}$. Set $\tau_{j+1}:=$ $\sigma_{1} \circ \sigma_{2} \circ \cdots \circ \sigma_{j+1}$. Then $\tau_{j+1}^{*}(\mathcal{J})$ is not divisible by $\mathcal{I}_{H_{1}^{j+1}}$ at $a_{j+1}$, and

$$
\tau_{j+1}^{*}(\mathcal{I})=\mathcal{I}_{H_{0}^{j+1}}^{j \mu_{H, a}(\underline{\mathcal{I}}) d} \cdot \mathcal{I}_{H_{1}^{j+1}}^{\mu_{H, a}(\underline{\mathcal{I}}) d} \cdot \tau_{j+1}^{*}(\mathcal{J}) .
$$

(Consider the formal expansion of the pullback of $f(x)=x_{1}^{\mu_{H, a}(\underline{\mathcal{I}}) d} g(x) \in \mathcal{I}$.)

Therefore, there exists $i \geq 1$ such that $\operatorname{ord}_{a_{i+1}} \tau_{i+1}^{*}(\mathcal{J})=\operatorname{ord}_{a_{j+1}} \tau_{j+1}^{*}(\mathcal{J})$, for all $j \geq i$. (We can take $i$ to be the least order of a monomial not divisible by $x_{1}$ in the Taylor expansions of a set of generators of $\mathcal{J}$ at a.) Clearly, if $j \geq i$, then

$$
\mu_{H, a}(\underline{\mathcal{I}})=\mu_{a_{j+1}}\left(\underline{\mathcal{I}}_{j+1}\right)-\mu_{a_{j}}\left(\underline{\mathcal{I}}_{j}\right),
$$

so the result follows from Theorem 6.1.

Remark 6.3. The $\mu_{H, a}(\underline{\mathcal{I}})$ are not invariants of the weak equivalence class of $\underline{\mathcal{I}}$ [BM5, Example 5.14]. The latter example shows that Step II of our proof in Section 5 is not functorial with respect to weak equivalence classes.

\section{$\S 7 . \quad$ Passage from Local to Global}

In this section, we give two different proofs of Claim 5.1 in Step I.A in Section 5 (and of the analogous claim in Step I.B). The first proof is the method described by Kollár [Ko], which we present briefly here. (See [Ko, Thm. 105] for an axiomatization of the procedure.) The second proof is the method of [BM4], [BM5], which involves proving a stronger result than Theorem 1.3: we give an algorithm for functorial resolution of singularities, where each successive centre of blowing up is the maximum locus of a desingularization invariant. We use the notation of Section 4.

The invariant is essentially already present in the proof of Theorem 1.3 in Section 5; we just make it explicit below. The invariant is defined using a sequence of pairs - it begins with $(\operatorname{ord} \mathcal{N}(\underline{\mathcal{I}}), s)$ and the following pairs are determined in decreasing dimension, by induction. (The first pair is the same as that denoted $(d, s)$ in our first version of resolution of singularities [BM1, $\S 4]$.) 


\section{$\S 7.1 . \quad$ First proof}

For simplicity, assume that $N$ has constant dimension $n$. Choose a finite open covering $M=\cup U_{i}$ such that, for each $i$, we have a coefficient ideal $\underline{\mathcal{C}}_{U_{i}}(\underline{\mathcal{I}})$ as in Step I.A. Set $M^{\prime}:=\coprod_{i} U_{i}$ (the disjoint union) and let $\gamma: M^{\prime} \rightarrow M$ denote the natural morphism. ( $\gamma$ is smooth.) The fibre-product $M^{\prime \prime}:=M^{\prime} \times_{M} M^{\prime}$ can be identified with the disjoint union $\coprod_{i \leq j} U_{i} \cap U_{j}$, so that, if $\tau_{1}, \tau_{2}: M^{\prime \prime} \rightarrow M^{\prime}$ are the two projections, then $\left.\tau_{1}\right|_{U_{i} \cap U_{j}}$ and $\left.\tau_{2}\right|_{U_{i} \cap U_{j}}$ are the inclusions $U_{i} \cap U_{j} \hookrightarrow$ $U_{i}$ and $U_{i} \cap U_{j} \hookrightarrow U_{j}$ (respectively).

Set $\underline{\mathcal{I}}^{\prime}:=\gamma^{*}(\underline{\mathcal{I}})$ and $\underline{\mathcal{I}}^{\prime \prime}:=\tau_{1}^{*}\left(\underline{\mathcal{I}}^{\prime}\right)=\tau_{2}^{*}\left(\underline{\mathcal{I}}^{\prime}\right)$. The coefficient ideals $\underline{\mathcal{C}}_{U_{i}}(\underline{\mathcal{I}})$ induce a marked ideal $\underline{\mathcal{C}}^{\prime}$ of dimension $n-1$ on $M^{\prime}$. Set $\underline{\mathcal{C}}_{1}^{\prime \prime}:=\tau_{1}^{*}\left(\underline{\mathcal{C}}^{\prime}\right), \underline{\mathcal{C}}_{2}^{\prime \prime}:=$ $\tau_{2}^{*}\left(\underline{\mathcal{C}}^{\prime}\right)$. Then $\underline{\mathcal{C}}^{\prime}$ is equivalent to $\underline{\underline{I}}^{\prime}$, and $\underline{\mathcal{C}}_{1}^{\prime \prime}$ is equivalent to $\underline{\mathcal{C}}_{2}^{\prime \prime}$.

By the inductive assumption of functorial resolution of singularities in dimension $n-1$, there are resolution sequences associated to $\underline{\mathcal{C}}^{\prime}, \underline{\mathcal{C}}_{1}^{\prime \prime}$ and $\underline{\mathcal{C}}_{2}^{\prime \prime}$ such that: (1) the resolution sequences for $\underline{\mathcal{C}}_{1}^{\prime \prime}$ and $\underline{\mathcal{C}}_{2}^{\prime \prime}$ are the same; (2) the morphism $\tau_{1}$ (or $\tau_{2}$ ) lifts throughout the resolution sequences for $\underline{\mathcal{C}}_{1}^{\prime \prime}$ and $\underline{\mathcal{C}}^{\prime}$ (or $\underline{\mathcal{C}}_{2}^{\prime \prime}$ and $\left.\underline{\mathcal{C}}^{\prime}\right)$.

Since $\underline{\mathcal{C}}^{\prime}$ is equivalent to $\underline{\mathcal{I}}^{\prime}$, the resolution sequence for $\underline{\mathcal{C}}^{\prime}$ is a resolution sequence for $\underline{\mathcal{I}}^{\prime}$. Consider the centre $C_{0}^{\prime} \subset M_{0}^{\prime}:=M^{\prime}$ of the first blowing-up in this sequence. For each $i$, let $C_{0 i}^{\prime}$ denote the restriction of $C_{0}^{\prime}$ to $U_{i}$. Then $C_{0 i}^{\prime}\left|U_{i} \cap U_{j}=C_{0 j}^{\prime}\right| U_{i} \cap U_{j}$, for all $i \leq j$.

Therefore, the $C_{0 i}^{\prime}$ glue together to define a smooth closed subvariety $C_{0}$ of $M_{0}:=M$. We thus obtain the centre of the first global blowing-up, and can continue in the same way to get the entire functorial resolution sequence for $\underline{\mathcal{I}}$.

The same argument works for the analogous claim in Step I.B.

\section{$\S 7.2$. Second proof}

Given a marked ideal $\underline{\mathcal{I}}$, we will construct a resolution sequence and an invariant (with values in an ordered set) defined at the points of the $\operatorname{cosupp} \underline{\mathcal{I}}_{j}$ (where the $\underline{\mathcal{I}}_{j}$ are the successive transforms of $\underline{\mathcal{I}}$ ), such that the invariant is upper-semicontinuous, we blow up its maximum locus at each step, and the invariant decreases with (finitely many) blowings-up. The following theorem makes this precise. Let $\mathbb{Q}_{>0}$ (or $\mathbb{Q}_{\geq 0}$ ) denote the set of positive (or nonnegative) rational numbers.

Theorem 7.1. Let $\underline{\mathcal{I}}=(M, N, E, \mathcal{I}, d)$ denote a marked ideal, where $d>0$. Then $\underline{\mathcal{I}}$ admits a resolution of singularities

$$
M=M_{0} \stackrel{\sigma_{1}}{\longleftarrow} M_{1} \longleftarrow \cdots \stackrel{\sigma_{t}}{\longleftarrow} M_{t},
$$


such that, if $\underline{\mathcal{I}}_{j}$ denotes the $j$ 'th transform of $\underline{\mathcal{I}}$, then the following properties are satisfied.

(1) There are Zariski upper-semicontinuous functions $\operatorname{inv}_{\underline{\underline{\mathcal{I}}}}, \mu_{\underline{\underline{\mathcal{I}}}}$ and $J_{\underline{\underline{\mathcal{I}}}}$ defined on $\operatorname{cosupp} \underline{\mathcal{I}}_{j}$, for all $j$, where $\operatorname{inv}_{\underline{\mathcal{I}}}$ takes values in the set of sequences consisting of finitely many pairs in $\mathbb{Q}_{>0} \times \mathbb{N}$ followed by 0 or $\infty$ (ordered lexicographically), $\mu_{\underline{\underline{I}}}$ takes values in $\mathbb{Q}_{\geq 0} \cup\{\infty\}$, and $J_{\underline{\underline{I}}}$ takes values in the set of subsets of $E_{j}$, for all $j$ (ordered as in Step II.A in Section 5).

(2) Each centre of blowing up $C_{j} \subset M_{j}$ is given by the maximum locus of $\left(\operatorname{inv}_{\underline{\underline{\mathcal{I}}}}, J_{\underline{\mathcal{I}}}\right)$ on $\operatorname{cosupp} \underline{\mathcal{I}}_{j}$ (where pairs are ordered lexicographically).

(3) Let $a \in \operatorname{cosupp} \underline{\mathcal{I}}_{j+1}$ and $b=\sigma_{j+1}(a)$. If $b \notin C_{j}$, then

$$
\operatorname{inv}_{\underline{\mathcal{I}}}(a)=\operatorname{inv}_{\underline{\mathcal{I}}}(b), \quad \mu_{\underline{\mathcal{I}}}(a)=\mu_{\underline{\mathcal{I}}}(b), \quad J_{\underline{\mathcal{I}}}(a)=J_{\underline{\mathcal{I}}}(b) .
$$

If $b \in C_{j}$, then

$$
\left(\operatorname{inv}_{\underline{\underline{\mathcal{I}}}}(a), \mu_{\underline{\mathcal{I}}}(a)\right)<\left(\operatorname{inv}_{\underline{\mathcal{I}}}(b), \mu_{\underline{\mathcal{I}}}(b)\right) .
$$

(4) Suppose that $\varphi: M^{\prime} \rightarrow M$ is a smooth morphism and that $\underline{\mathcal{I}^{\prime}}=\left(M^{\prime}, N^{\prime}, E^{\prime}\right.$, $\left.\underline{\mathcal{I}}^{\prime}, d^{\prime}\right)$ is a marked ideal such that $\underline{\mathcal{I}}^{\prime}$ is equivalent to $\varphi^{*}(\underline{\mathcal{I}})$ (in particular, $\left.E^{\prime}=\varphi^{-1}(E)\right)$ and $\operatorname{dim} N^{\prime}=\operatorname{dim} \varphi^{-1}(N)$. Then $\varphi$ lifts to smooth morphisms $\varphi_{j}$ throughout the resolution towers for $\underline{\mathcal{I}}^{\prime}$ and $\underline{\mathcal{I}}$, and, for each $j$,

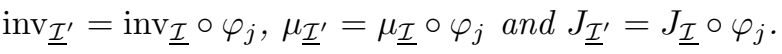

Proof. We follow the steps of the proof of Theorem 1.3 in Section 5.

\section{Step I. $\underline{\mathcal{I}}$ of maximal order.}

Case A. $E=\emptyset$. The coefficient ideals $\underline{\mathcal{C}}_{U}(\underline{\mathcal{I}})$ admit resolution sequences and invariants as required, by induction. Over each $U$, the desingularization of $\underline{\mathcal{I}}$ is realized by that of $\underline{\mathcal{C}}_{U}(\underline{\mathcal{I}})$. If $x$ is a point of $\operatorname{cosupp} \underline{\mathcal{I}}_{j}$ lying over $U$, we set

$$
\overline{\operatorname{inv}}_{\underline{\underline{I}}}(x):=\left(0, \operatorname{inv}_{\underline{\mathcal{C}}_{U}(\underline{\mathcal{I}})}(x)\right), \quad \bar{\mu}_{\underline{\underline{\mathcal{I}}}}(x):=\mu_{\underline{\mathcal{C}}_{U}(\underline{\mathcal{I}})}(x), \quad \bar{J}_{\underline{\mathcal{I}}}(x):=J_{\underline{\mathcal{C}}_{U}(\underline{\mathcal{I}})}(x) .
$$

Case B. General maximal order case. The marked ideal $\underline{\mathcal{J}}$ (defined using $\underline{\mathcal{C}}_{U}\left(\underline{\mathcal{I}}_{\emptyset}\right)$ ) has empty exceptional divisor. We set

$$
\overline{\operatorname{inv}}_{\underline{\mathcal{I}}}(x):=\left(s, \operatorname{inv}_{\underline{\mathcal{I}}}(x)\right), \quad \bar{\mu}_{\underline{\mathcal{I}}}(x):=\mu_{\underline{\mathcal{I}}}(x), \quad \bar{J}_{\underline{\underline{\mathcal{I}}}}(x):=J_{\underline{\mathcal{I}}}(x),
$$

for $x \in \operatorname{cosupp} \underline{\mathcal{J}}_{j}$, until $\underline{\mathcal{J}}$ is resolved. If the sequences of blowings-up of the $\underline{\mathcal{J}}$ do not resolve $\underline{\mathcal{I}}$, then we repeat the process using the new $s=\max s(x)$, as in Section 5. 
Note that if $x \in \operatorname{cosupp} \underline{\mathcal{I}}_{j}$ and $s(x)<s$, then all blowings-up in the desingularization tower of $\underline{\mathcal{I}}$ are isomorphisms over $x$, until we reach a year $q_{k}$ where $s(x)$ is the maximum value. Then the values of the invariants at $x$ equal their values in year $q_{k}$ (by definition; see also Step II.B below).

Step II. General $\underline{\mathcal{I}}$. First suppose that $\mathcal{I}=0$. If $x \in \operatorname{cosupp} \underline{\mathcal{I}}=N$, then we set

$$
\operatorname{inv}_{\underline{\mathcal{I}}}(x):=\infty, \quad \mu_{\underline{\mathcal{I}}}(x):=\infty, \quad J_{\underline{\mathcal{I}}}(x):=\emptyset .
$$

We blow up with centre $N$ to resolve singularities.

Now suppose that $\mathcal{I} \neq 0$.

Case A. Monomial case. If $x \in \operatorname{cosupp} \underline{\mathcal{I}}_{j}$, then we set

$$
\operatorname{inv}_{\underline{\underline{I}}}(x):=0, \quad \mu_{\underline{\underline{I}}}(x):=\mu_{x}\left(\underline{\mathcal{I}}_{j}\right)=\frac{\operatorname{ord}_{x} \mathcal{I}_{j}}{d},
$$

and we let $J_{\underline{\mathcal{I}}}(x)$ denote the maximum among the subsets of $E_{j}$ that define the components of $\operatorname{cosupp} \underline{\mathcal{I}}_{j}$ at $x$. The required properties in this case have been proved in Section 4.

Case B. General case. We resolve the singularities of the companion ideal $\underline{\mathcal{G}}:=\underline{\mathcal{G}}(\underline{\mathcal{I}})$ (which is of maximal order) in order to reduce the maximum order of the ideal $\mathcal{N}(\underline{\mathcal{I}})$. If $x \in \operatorname{cosupp} \underline{\mathcal{G}}_{j}$, then we set

$$
\operatorname{inv}_{\underline{\underline{\mathcal{I}}}}(x):=\left(\frac{\operatorname{ord} \mathcal{N}(\underline{\mathcal{I}})}{d}, \overline{\operatorname{inv}}_{\underline{\mathcal{G}}}(x)\right), \quad \mu_{\underline{\mathcal{I}}}(x):=\bar{\mu}_{\underline{\mathcal{G}}}(x), \quad J_{\underline{\underline{\mathcal{I}}}}(x):=\bar{J}_{\underline{\mathcal{G}}}(x) .
$$

If $x \in \operatorname{cosupp} \underline{\mathcal{I}} \backslash \operatorname{cosupp} \underline{\mathcal{G}}$, then $\operatorname{ord}_{x} \mathcal{N}(\underline{\mathcal{I}})$ will be the maximum order of $\mathcal{N}(\underline{\mathcal{I}})$ in some neighbourhood of $x$, so we can define the invariant in the same way over such a neighbourhood. (When we resolve the singularities of $\underline{\mathcal{G}}(\underline{\mathcal{I}})$, either $\operatorname{cosupp} \underline{\mathcal{I}}_{r_{1}}=\emptyset$ or ord $\mathcal{N}\left(\underline{\mathcal{I}}_{r_{1}}\right)<\operatorname{ord} \mathcal{N}(\underline{\mathcal{I}})$ after $r_{1}$ steps, and we repeat the process using $\left(\mathcal{N}\left(\underline{\mathcal{I}}_{r_{1}}\right)\right.$, ord $\left.\mathcal{N}\left(\underline{\mathcal{I}}_{r_{1}}\right)\right)$. If $x \in \operatorname{cosupp} \underline{\mathcal{G}}\left(\underline{\mathcal{I}}_{r_{1}}\right)$ maps to the complement of $\operatorname{cosupp} \underline{\mathcal{G}}(\underline{\mathcal{I}})$, then all previous blowings-up are isomorphisms at the successive images of $x$, so the values of the invariants at these points are the same as at $x$.)

All properties required in the theorem follow by induction and semicontinuity of $\operatorname{ord}_{x}$.

\section{§8. Comparison of Algorithms and an Example}

In this final section, we prove Corollary 1.4 and we give an example to show that the versions of canonical desingularization (Theorem 1.1) in [EV2], [W], [Ko] involve blowings-up with centres that are not necessarily smooth (see also [Ma, Ex. 11-5]). 


\section{§8.1. Włodarczyk’s method}

Let $\underline{\mathcal{I}}=(M, N, E, \mathcal{I}, d)$ denote a marked ideal. In the case that $\underline{\mathcal{I}}$ is of maximal order, Włodarczyk introduces a homogenized ideal $\underline{\mathcal{H}}(\underline{\mathcal{I}})=(M, N, E$, $\mathcal{H}(\underline{\mathcal{I}}), d)$, where

$$
\mathcal{H}(\underline{\mathcal{I}}):=\sum_{j=0}^{d-1} \mathcal{D}^{j}(\mathcal{I}) \cdot\left(\mathcal{D}^{d-1}(\mathcal{I})\right)^{j} .
$$

Then $\underline{\mathcal{H}}(\underline{\mathcal{I}})$ is weak-equivalent to $\underline{\mathcal{I}}$ [W, Lemma 3.5.2]. In general, $\underline{\mathcal{H}}(\underline{\mathcal{I}})$ is not equivalent to $\underline{\mathcal{I}}$. It follows from Corollary 3.6, however, that if $E=\emptyset$, then $\underline{\mathcal{C}}^{k}(\underline{\mathcal{I}})$ and $\underline{\mathcal{H}}(\underline{\mathcal{I}})$ are both equivalent to $\underline{\mathcal{I}}$. (The same statements hold for the variant $\underline{\mathcal{W}}(\underline{\mathcal{I}})$ of $\underline{\mathcal{H}}(\underline{\mathcal{I}})$ introduced by Kollár $[\mathrm{Ko}]$.)

Consider the desingularization algorithm as described in Section 5. As we said in Remark 1.5, we will ignore the small difference in the way that Włodarczyk treats Step I.B. The essential difference in his proof is his use of the homogenized ideal. Instead of using the ideal $\underline{\mathcal{C}}_{E}^{d-1}(\underline{\mathcal{I}})$ of $\S 3.4$, he uses $\underline{\mathcal{C}}^{d-1}(\underline{\mathcal{H}}(\underline{\mathcal{I}}))$. (See Remark 3.13.) Following the algorithm as presented in Section 5 , this introduces a change in Step I, where, instead of our coefficient ideal $\underline{\mathcal{C}}_{\emptyset, P}(\underline{\mathcal{I}})$ (see (4.1)), Włodarczyk would use

$$
\underline{\mathcal{C}}_{P}(\underline{\mathcal{I}}):=\left(U, P, \emptyset,\left.\mathcal{C}^{d-1}(\underline{\mathcal{H}}(\underline{\mathcal{I}}))\right|_{P}, d_{\underline{\mathcal{C}}}\right) .
$$

(Likewise in Kollár's version, using $\underline{\mathcal{W}}(\underline{\mathcal{I}})$.)

A priori, this change might result in a change in the actual recipe for choosing the centres of blowing up. However, since $\underline{\mathcal{C}}^{d-1}(\underline{\mathcal{H}}(\underline{\mathcal{I}}))$ is equivalent to $\underline{\mathcal{I}}$ when $E=\emptyset$, it follows that $\underline{\mathcal{C}}_{P}(\underline{\mathcal{I}})$ is equivalent to $\underline{\mathcal{C}}_{\emptyset, P}(\underline{\mathcal{I}})$.

Equivalence of the companion ideal (Sect. 5, Step II.B) to the variants used in $[\mathrm{W}]$ and $[\mathrm{Ko}]$ follows using Corollary 5.3. We thus obtain Corollary 1.4.

\section{§8.2. Example}

Let $X$ denote the subvariety of $\mathbb{A}^{4}$ defined by the ideal $\mathcal{I}$ generated by $y^{2}-x^{3}$ and $x^{4}+x z^{2}-w^{3}$. We consider resolution of singularities (Section 5) of the corresponding marked ideal $\underline{\mathcal{I}}=\left(\mathbb{A}^{4}, \mathbb{A}^{4}, \emptyset, \mathcal{I}, 1\right)$ (which we write simply as $(\emptyset, \mathcal{I}, 1))$. Then the companion ideal $\underline{\mathcal{G}}(\underline{\mathcal{I}})=\underline{\mathcal{N}}(\underline{\mathcal{I}})=(\emptyset, \mathcal{I}, 2)$ (Step II.B). The ideal $\mathcal{I}$ has maximum order 2 precisely at the origin, so that $C_{0}:=\{0\}$ is the centre of the first blowing-up $\sigma_{1}$, by Step I.A. Let $\underline{\mathcal{I}}_{1}=\left(E_{1}, \mathcal{I}_{1}, 1\right)$ denote the transform of $\underline{\mathcal{I}}$ by $\sigma_{1}$. In the $x$-chart of $\sigma_{1}, E_{1}$ is given by $(x)$ and

$$
\mathcal{N}\left(\underline{\mathcal{I}}_{1}\right)=\mathcal{N}(\underline{\mathcal{I}})_{1}=\left(x-y^{2}, x\left(x+z^{2}-w^{3}\right)\right)=\left(x-y^{2}, y^{2}\left(y^{2}+z^{2}-w^{3}\right)\right) ;
$$


thus $\mathcal{N}\left(\underline{\mathcal{I}}_{1}\right)$ has maximal order 1. According to Step I.B, the centre $C_{1}$ of the next blowing-up $\sigma_{2}$ is given by $x=y=0$.

But the ideal of the strict transform $X_{1}$ of $X$ in the $x$-chart above is generated by

$$
x-y^{2}, \quad y^{2}+z^{2}-w^{3} .
$$

Therefore, $C_{1} \cap X_{1}$ is given by

$$
x=y=0, \quad z^{2}-w^{3}=0 .
$$

Moreover, Sing $X_{1}=\{0\}$.

\section{References}

[Be] B. M. Bennett, On the characteristic functions of a local ring, Ann. of Math. (2) 91 (1970), 25-87.

[BM1] E. Bierstone and P. D. Milman, Semianalytic and subanalytic sets, Inst. Hautes Études Sci. Publ. Math., No. 67 (1988), 5-42.

[BM2] U Uniformization of analytic spaces, J. Amer. Math. Soc. 2 (1989), no. 4, 801-836.

[BM3] A simple constructive proof of canonical resolution of singularities, in Effective methods in algebraic geometry (Castiglioncello, 1990), 11-30, Progr. Math., 94, Birkhäuser, Boston, MA, 1991.

[BM4] Canonical desingularization in characteristic zero by blowing up the maximum strata of a local invariant, Invent. Math. 128 (1997), no. 2, 207-302.

[BM5] , Desingularization algorithms. I. Role of exceptional divisors, Mosc. Math. J. 3 (2003), no. 3, 751-805.

[BM6] ( Resolution of singularities in Denjoy-Carleman classes, Selecta Math. (N.S.) 10 (2004), no. 1, 1-28.

[C] V. Cossart, Forme normale d'une fonction sur un $k$-schéma de dimension 3 et de caractéristique positive, in Géométrie algébrique et applications, I (La Rábida, 1984), 1-21, Hermann, Paris, 1987.

[EV1] S. Encinas and O. Villamayor, Good points and constructive resolution of singularities, Acta Math. 181 (1998), no. 1, 109-158.

[EV2] _ A new proof of desingularization over fields of characteristic zero, Rev. Mat. Iberoamericana 19 (2003), no. 2, 339-353.

[EV3] L Rees algebras and resolution of singularities, preprint (2007), math.AG/0702836.

[G] J. Giraud, Sur la théorie du contact maximal, Math. Z. 137 (1974), 285-310.

[Ha] R. Hartshorne, Algebraic geometry, Springer, New York, 1977.

[Hi1] H. Hironaka, Resolution of singularities of an algebraic variety over a field of characteristic zero. I, II, Ann. of Math. (2) 79 (1964), 109-203; ibid. (2) 79 (1964), 205-326.

[Hi2] _ Idealistic exponents of singularity, in Algebraic geometry (J. J. Sylvester Sympos., Johns Hopkins Univ., Baltimore, Md., 1976), 52-125, Johns Hopkins Univ. Press, Baltimore, Md., 1977.

[Hi3] _ Theory of infinitely near singular points, J. Korean Math. Soc. 40 (2003), no. 5, 901-920.

[Hi4] H. Hironaka, A program for resolution of singularities, in all characteristics $p>0$ and in all dimensions, preprint (2006). 
[Ka] H. Kawanoue, Toward resolution of singularities over a field of positive characterstic. Part I. Foundation; the language of the idealistic filtration, preprint (2006), math.AG/0607009.

[Ko] J. Kollár, Lectures on resolution of singularities, Ann. of Math. Stud., 166, Princeton Univ. Press, Princeton, NJ, 2007.

[Ma] K. Matsuki, Notes on the inductive algorithm of resolution of singularities by S. Encinas and O. Villamayor, math.AG/0103120.

[V1] O. Villamayor, Constructiveness of Hironaka's resolution, Ann. Sci. École Norm. Sup. (4) 22 (1989), no. 1, 1-32.

[V2] , Patching local uniformizations, Ann. Sci. École Norm. Sup. (4) 25 (1992), no. $6,629-677$.

[W] J. Włodarczyk, Simple Hironaka resolution in characteristic zero, J. Amer. Math. Soc. 18 (2005), no. 4, 779-822. 